\title{
4 Moleküle - Bindungen und Reaktionen
}

\author{
Nikolaus Risch
}

\subsection{Chemische Bindungen}

\subsubsection{Chemische Formeln}

Die moderne Sprache der molekularen Physik basiert auf Symbolen, die im Laufe von Jahrhunderten entwickelt wurden. Bereits in der Alchimie des Mittelalters wurden derartige Symbole für chemische und pharmazeutische Arbeitstechniken verwendet. Diese dienten jedoch meist dazu, den Zugang zu der Geheimwissenschaft Alchimie zu erschweren. Die heute verwendete Symbolik strebt das Gegenteil an, nämlich die komplexen Strukturen der Natur möglichst einfach und unabhängig von Sprachbarrieren verständlich und zugleich exakt darzustellen. Richtlinien hierzu werden von Chemikern in der International Union of Pure and Applied Chemistry (IUPAC) entwickelt - dem Pendant zur IUPAP [1]. Grundlegendes Element einer chemischen Formel ist die Tatsache, da $B$ Buchstaben (für jedes Element ein eigenes Buchstabensymbol: C für Kohlenstoff, H für Wasserstoff, O für Sauerstoff usw.) durch Striche miteinander verbunden, eine Aussage über die Substanz mit all ihren beobachtbaren Eigenschaften macht bzw. machen soll.

Abhängig von der Zielsetzung werden für ein Molekül, wie beispielsweise das Ethanol, z.T. deutlich unterschiedliche Darstellungsarten von Formeln verwendet (Abb. 4.1; $a-f$ ). So berücksichtigen a und $a^{\prime}$ die elementare Zusammensetzung ( $a^{\prime}$ zusätzlich die Funktionalität der OH-Gruppe), $b$ und $\mathrm{c}$ auch die Konstitution und $\mathrm{d}$

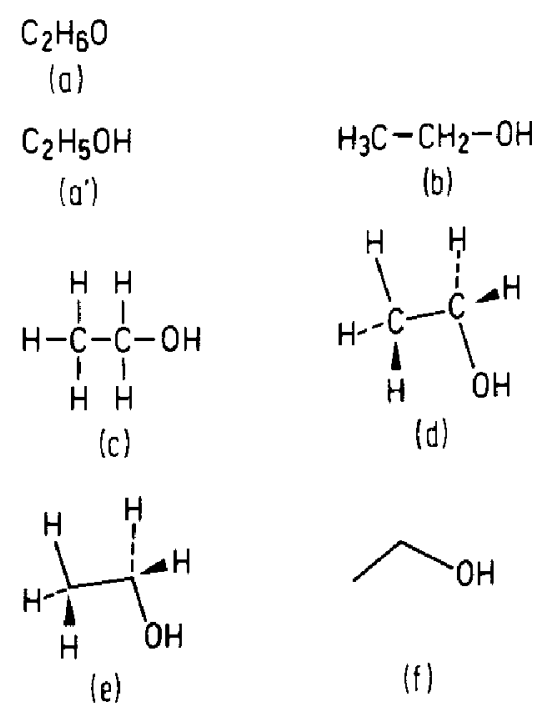

Abb.4.1 Unterschiedliche Möglichkeiten für die Wiedergabe der Struktur des Ethanols. 
und e darüber hinaus den dreidimensionalen (sterischen) Aufbau der Moleküle. Als vereinfachte Schreibweise wird wegen der besseren Übersichtlichkeit in der modernen Organischen Chemie der Kohlenstoff nur noch als Eck- bzw. Endpunkt des Linienzuges verstanden, der das Kohlenstoffgerüst verbindet (e-f). Darüber hinaus verzichtet man gern auf die Abbildung der Wasserstoffatome, die an den Kohlenstoff gebunden sind (f). Da der Kohlenstoff vierbindig ist (tetraedrische Umgebung), füllt man dann gedanklich jede Kohlenstoffposition mit der Anzahl an Wasserstoffatomen auf, die eine Vierbindigkeit gewährleistet (gilt entsprechend auch bei Mehrfachbindungen des Kohlenstoffs).

\subsubsection{Die Periodizität chemischer Eigenschaften}

Die meisten Eigenschaften der Elemente wechseln in periodischer Weise, die dadurch deutlich wird, indem man sie nach ihrer Ordnungszahl (Anzahl der Protonen) anordnet.

Diese entscheidende Beobachtung eröffnete die ungemein wichtige Möglichkeit gezielter Forschung auf der Grundlage vorhersagbarer Charakteristika. Wie sieht diese Welt der Chemie aus und wie hat sie sich entwickelt?

Schon relativ früh lernte man zwischen Metallen und Nichtmetallen zu unterscheiden. Die Entwicklung zu den Döbereinerschen Triaden (Tab. 4.1) ließ weitere Zusammenhänge deutlich werden und führte schließlich über Newlands Oktavengesetz (kannte keine Edelgase) zum immer noch modernen Periodensystem der Elemente (PSE), das in den entscheidenen Punkten 1869 von D. Mendelejeff bzw. L. Meyer aufgestellt wurde.

Die anfängliche Anordnung nach relativen Atommassen ließ jedoch bald einige Unstimmigkeiten erkennen. Moseley konnte dann belegen, daß die Ordnungszahl (Kernladung) das geeignete Kriterium für die Erklärung des chemischen Verhaltens und damit der Einordnung in das PSE ist. Er zeigte, da $\$$ die nach Bestrahlung mit energiereichen Elektronen von den Elementen emittierte Röntgenstrahlung mit ihrer Ladung korreliert und Informationen über die Energieniveaus der Elektronen in dem betreffenden Atom enthält.

Ordnet man nun die Elemente nach ihrer Ordnungszahl, dann wiederholen sich bestimmte chemische und physikalische Eigenschaften in charakateristischen Intervallen, und man erhält das PSE in seiner heute bekannten Form (s. hinterer Einbanddeckel). Die 107 Elemente werden in sieben Perioden angeordnet, so daß chemisch ähnliche Elemente in senkrechten Spalten untereinander stehen (2 Elemente in der ersten Periode, 8 in der zweiten und dritten, 18 in der vierten und fünften und 32 in der sechsten). Auf diese Weise ergibt sich beispielsweise in der ersten Spalte die Gruppe der Alkalimetalle ( $\mathrm{Li}, \mathrm{Na}, \mathrm{K}, \mathrm{Rb}, \mathrm{Cs}$ und $\mathrm{Fr}$ ), die alle im Zuge ihrer ausgeprägten Reaktivität (niedere Ionisationsenergie) dazu neigen, einfach positiv geladene Ionen zu bilden. Die chemisch weitgehend inerten Edelgase ( $\mathrm{He}, \mathrm{Ne}, \mathrm{Ar}, \mathrm{Kr}, \mathrm{Xe}, \mathrm{Rn}$ ) stehen hingegen jeweils am Ende einer Periode. Das PSE bietet einfache und schnelle Unterscheidungsmöglichkeiten zwischen Metallen und Nichtmetallen an und erlaubt relative Voraussagen zur Elektronegativität, zum Atomradius und zu weiteren wichtigen Eigenschaften.

In der kompakten Darstellung (Abb. 4.2) bietet das PSE eine deutliche und übersichtliche Einteilung in drei Kategorien: 
a) Hauptgruppenelemente mit z.T. extrem unterschiedlichen Eigenschaften: Hier unterscheiden sich die Elemente einer Zeile durch die Zahl der s- und p-Elektronen in der äußeren Schale (Valenzelektronen).

b) Nebengruppenelemente (Übergangsmetalle) mit durchaus ähnlichen, aber deutlich unterscheidbaren Eigenschaften: Hier werden die d-Elektronen der nächstinneren Schale aufgefüllt, deren Orbitale dichter am Kern liegen als die der äußersten s- und p-Elektronen.

c) Innere Übergangsmetalle (Lanthanoide, Actinoide) mit nahezu gleichen Eigenschaften: Hier werden die f-Elektronen in einer noch weiter innen liegenden Schale aufgefüllt, die jedoch das chemische Verhalten kaum beeinflussen.

Tab.4.1 Beschreibung der Döbereinerschen Triaden.

\begin{tabular}{llll}
\hline Triaden- & Elementare & Hauptverbindungen & Spezielle Eigenschaften \\
elemente & Form & & \\
und relative & & \\
Atommassen & &
\end{tabular}

$\mathrm{Cl}$; $\mathrm{Br}$; I Farbige, Bilden einfache Salze, die Freie Elemente reagieren

35,5; 80; 127 zweiatomige (-1)-Ionen enthalten: $\mathrm{Cl}^{-}$, heftig mit Elektronendonato-

$\mathrm{Cl}_{2}$ (gelbgrün)

$\mathrm{Br}_{2}$ (braun)

$\mathrm{I}_{2}$ (violett)

$\mathrm{Br}^{-}, \mathrm{I}^{-}$. Bilden mit Sauer-

ren unter Bildung negativer

stoff Ionen der Ladung

Ionen: $\mathrm{Cl}^{-}, \mathrm{Br}^{-}, \mathrm{I}^{-}$:

$(-1)$, die ein bis vier

$2 \mathrm{Na}+\mathrm{Cl}_{2} \rightleftarrows 2 \mathrm{Na}^{+}+2 \mathrm{Cl}^{-}$

Sauerstoffatome enthalten:

$\mathrm{ClO}_{4}^{-}, \mathrm{ClO}_{3}^{-}, \mathrm{ClO}^{-}$, $\mathrm{BrO}_{3}^{-}, \mathrm{IO}_{3}^{-}, \mathrm{IO}_{4}^{-}$.

Wasserstoffverbindungen

sind: $\mathrm{HCl}, \mathrm{HBr}, \mathrm{HI}$.

$\mathrm{I}_{2}+\mathrm{S}^{2-} \rightleftarrows 2 \mathrm{I}^{-}+\mathrm{S}$

Salze (wie $\mathrm{NaCl}$ ) lösen sich

sehr gut in Wasser. Haloge-

nide von $\mathrm{Li}, \mathrm{Na}$ und $\mathrm{K}$ erge-

ben neutrale Lösungen. Was-

serstoffverbindungen sind

starke Säuren und dissoziie-

ren in Wasser vollständig:

$\mathrm{HBr}+\mathrm{H}_{2} \mathrm{O} \rightleftarrows \mathrm{H}_{3} \mathrm{O}^{+}+\mathrm{Br}$

$\mathrm{Ca} ; \mathrm{Sr} ; \mathrm{Ba} \quad$ Reaktions-

$40 ; 88 ; 137$ freudige

Bilden Salze, die (+ 2)-

Salze geben in der Flamme

Metalle

Ionen enthaiten: $\mathrm{Ca}^{2+}$,

$\mathrm{Sr}^{2+}, \mathrm{Ba}^{2+}$ in $\mathrm{BaSO}_{4}$,

kräftige Farben: $\mathrm{Ca}$ (orange).

Sr(rot), Ba (grün). Sulfate

$\mathrm{CaCO}_{3}, \mathrm{SrCl}_{2}$ usw.

und Carbonate sind unlöslich.

Metalle verdrängen langsam

Wasserstoff aus Wasser.

Li; Na; K

$7 ; 23 ; 39$

Sehr reaktions-

Bilden Salze, die $(+1)-$

Fast alle Salze sind löslich:

freudige Metalle

$\mathrm{K}^{+}$in $\mathrm{NaCl}, \mathrm{Li}_{2} \mathrm{CO}_{3}$,

$\mathrm{K}_{3} \mathrm{PO}_{4}$ usw.

Metalle und Salze ergeben

kräftig gefärbte Flammen: Li

(rot), Na (gelb), K (purpur).

Metalle reagieren heftig mit

Wasser unter Bildung von

Wasserstoff und löslichen io-

nischen Hydroxiden:

$2 \mathrm{Na}+2 \mathrm{H}_{2} \mathrm{O}$

$$
\rightleftarrows \mathrm{H}_{2}+2 \mathrm{Na}^{+}+2 \mathrm{HO}^{-}
$$




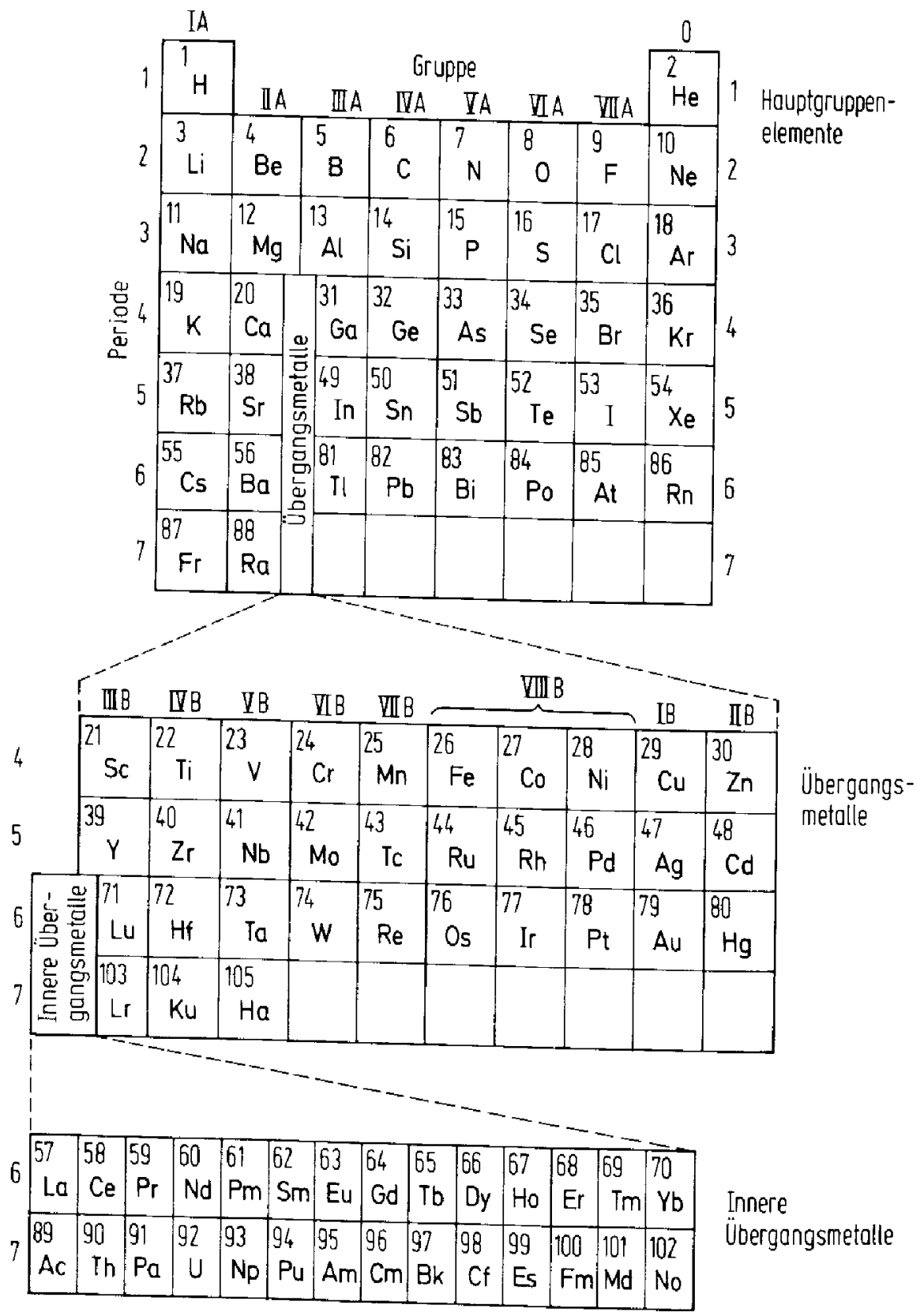

Abb. 4.2 Diese kompakte Form des Periodensystems betont die natürliche Einteilung der Elemente in drei Kategorien: Die extrem unterschiedlichen Hauptgruppenelemente, die sich stärker ähnelnden Übergangsmetalle und die einander ganz ähnlichen inneren Übergangsme-
talle.

\subsubsection{Metalle}

Atome gleichartiger und verschiedener Elemente können untereinander Bindungen eingehen. Diese werden bestimmt durch das Gleichgewicht der Coulomb'schen Kräfte der Elektronen und Protonen (Neutronen sind weitgehend chemische Zuschauer) unter Berücksichtigung des Pauli-Prinzips. Es kommt so zu größeren Verbänden, Molekülen, Kristallen etc.. Aber keineswegs jede denkbare Kombination von Atomen ergibt eine stabile Verbindung. Die entscheidende Voraussetzung zum Verständ- 
nis der zunächst empirischen Regeln, welche Kombinationen von Atomen zu faßbaren Produkten führen, vermittelt die Theorie der chemischen Bindung.

Die Mehrzahl aller Elemente zählt man zu den Metallen. Wie erklären sich nun die allgemein bekannten Charakteristika von Metallen, wie metallischer Glanz, hohe elektrische Leitfähigkeit und Wärmeleitfähigkeit, gute Verformbarkeit (Schmiedekunst), relativ hohe Dichte, hoher Schmelzpunkt etc.? Metalle kristallisieren in der Regel in dichtest gepackten Strukturen und bestehen aus praktisch unendlichen Anordnungen von aneinander gebundenen Atomen, wobei jedes Atom - im Gegensatz zu den Nichtmetallen - eine hohe Koordinationszahl (häufig 8 oder 12) besitzt. Sie weisen stets viel mehr atomare Valenzorbitale auf, als sie Valenzelektronen zur Besetzung dieser Orbitale zur Verfügung haben. Metallkristalle verhalten sich so, als ob sich ihre Valenzelektronen relativ frei innerhalb des Kristallgitters bewegen könnten. Man vergleicht die Elektronen hier gern mit einem See von negativen Ladungen, der die Atome in dieser Metallbindung fest zusammenhält.

Ein detailliertes Modell für die metallische Bindung liefert die Betrachtungsweise, in der ein Metallstück insgesamt als ein Riesenmolekül angesehen wird und delokalisierte Molekülorbitale sich über das gesamte Metallvolumen erstrecken. In der Orbitaltheorie (vgl. Abschn. 4.1.5) ergeben sich dann aus eng benachbarten Energieniveaus sogenannte Elektronenbänder, die teilweise besetzt sind und bei der Leitung des elektrischen Stroms genutzt werden (Abb. 4.3).

Metalle kommen in der Natur selten gediegen vor (Edelmetalle), sondern z. B. in Form oxidischer bzw. sulfidischer Verbindungen, den Erzen. Reduktive Behandlung der Erze setzt häufig die begehrten Metalle frei: Typisches Beispiel ist die Gewinnung von Eisen bzw. Stahl aus oxidischen Eisenerzen mit Kohle als Reduktionsmittel (HochofenprozeB; vgl. Band VI, Kap. 5).

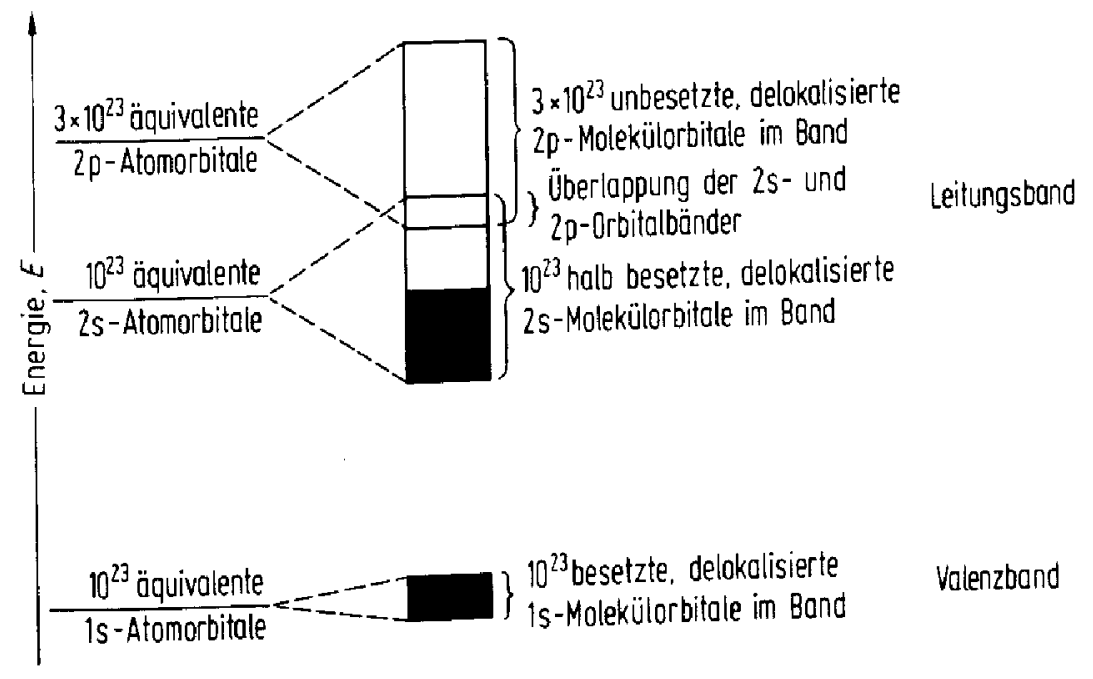

Abb.4.3 Delokalisierte Metallorbitalbänder im Lithium. Die ursprünglichen 2s- und 2pAtomorbitale liegen energetisch so dicht beieinander, $\mathrm{daB}$ sich die aus ihnen resultierenden Molekül- oder Metallorbitalbänder überlappen. Lithium besitzt ein Elektron für jedes $2 \mathrm{~s}$ Atomorbital und weist somit nur halb so viele Elektronen auf, wie in den 2s-Atomorbitalen bzw. dem delokalisierten 2s-Molekülorbitalband untergebracht werden können. Es ist somit nur eine infinitesimal kleine Energie erforderlich, um ein Elektron anzuregen, in den nächsthöheren (unbesetzten) Energiezustand überzugehen. Lithium ist demnach ein Elektrizitätsleiter. 


\subsubsection{Ionenbindung}

Neben der metallischen Bindung diskutiert man in der Chemie zwei weitere Haupttypen der chemischen Bindung. Es handelt sich um die heteropolare Ionenbindung und die homöopolare kovalente Bindung, wobei die Realität mehr oder weniger stark von diesen idealisierten Bindungstypen abweicht und es meist zu Mischformen kommt.

Metalle stehen im Periodensystem links und unten, und ihre Atome weisen nur wenige Valenzelektronen auf. Innerhalb einer Periode nimmt der metallische Charakter nach rechts stark ab, gleichzeitig steigt beim Übergang zu den typischen Nichtmetallen (oben rechts im PSE) die Elektronegativität (ungemein nützliche, empirisch ermittelte Größe, die die Tendenz angibt, eine Bindung durch Elektronen-Transfer zu begünstigen), d.h. die Ionenbindung ist das Resultat der elektrostatischen Anziehung von entgegengesetzt geladenen Teilchen, den Ionen.

Als einfache Regel kann man sich merken: Elemente, die im PSE weit voneinander entfernt sind (Elektronegativitätsunterschiede $>1.6$ ), neigen zur Ionenbindung ( $\mathrm{Re}$ doxprozesse, Abschn. 4.2.4), wobei es das Oktettprinzip ist, das die Natur der auftretenden Ionen bestimmt: Die klassischen Theorien der chemischen Bindung beruhen auf dem Bestreben der Atome, sich mit acht Valenzelektronen (8 Elektronen auf der äußeren Schale bzw. zwei Elektronen bei der Heliumkonfiguration) zu umgeben. Diese Elektronenkonfiguration ist besonders stabil und wird als Edelgaskonfiguration bezeichnet. Die besondere Stabilität äußert sich beispielsweise in der Tatsache, daß die Polarisierbarkeit bei den Edelgasen ein deutliches Minimum zeigt, so da $\beta$ diese Elektronenkonfigurationen keine besondere Neigung zeigen, sich auf einen äuBeren Einfluß von Feldern einzustellen und durch Bindungsbildung ein tieferes Potentialminimum zu bilden. Spektroskopisch sind diese Edelgaskonfigurationen dadurch charakterisiert, da $B$ der erste angeregte elektronische Zustand bei hohen Energien liegt.

Die Oktettregel verlangt das „Auffüllen bzw. Entleeren der äußeren Schale“. Das bedeutet für die Elemente der ersten Langperiode ( $\mathrm{Li}, \mathrm{Be}, \mathrm{B}, \mathrm{C}, \mathrm{N}, \mathrm{O}, \mathrm{F})$, daß sie jeweils so viele Valenzelektronen abgeben müssen, bis sie die Elektronenkonfiguration des $\mathrm{He}\left(1 \mathrm{~s}^{2}\right)$ erreicht haben, bzw. Elektronen aufnehmen müssen, bis sie die des $\mathrm{Ne}\left(2 \mathrm{~s}^{2} \mathrm{p}^{6}\right.$, äußere Schale) bilden. So erreicht das Lithiumatom durch Abgabe und das Fluoratom durch Aufnahme je eines Elektrons die Elektronenkonfiguration des Heliums bzw. des Neons:

$$
\mathrm{Li} \cdot+\left.\overline{\mathbf{F}}\left|\rightarrow \mathrm{Li}^{+}+\right| \overline{\mathbf{F}}\right|^{-}
$$

Die Ionenbindung beruht auf der elektrostatischen Anziehung entgegengesetzt geladener Ionen, die nach dem oben genannten Prinzip aus den Atomen entstehen. Zur Bildung der positiv geladenen Kationen neigen insbesondere Elemente mit geringer Zahl an Valenzelektronen (z. B. Metalle). Die negativ geladenen Anionen werden insbesondere von den Elementen der VI. und VII. Hauptgruppe des PSE erzeugt, die eine vergleichsweise hohe Elektronenaffinität (Elektronegativität) aufweisen. Bei komplexen Ionen können Metalle und Nichtmetalle als Zentralatome auftreten: $\mathrm{SO}_{4}{ }^{2-}, \mathrm{MnO}_{4}{ }^{-}, \mathrm{NH}_{4}{ }^{+},\left[\mathrm{Cu}\left(\mathrm{NH}_{3}\right)_{4}\right]^{2+}$.

Da die Wechselwirkungskräfte der Ionen isotrop sind, ist die Ionenbindung nicht gerichtet. Daraus resultieren hohe Koordinationszahlen, die im festen Zustand die Ausbildung von ,riesigen“ Strukturen aus Anionen und Kationen ermöglichen, den 
Ionenkristall. Die Anordnung der Ionen erfolgt hier in der Weise, daß die Gesamtwechselwirkungsenergie einen minimalen Wert annimmt. Koordinationszahlen und Abstand der Ionen im Gitter (Gittertyp) werden vor allem durch die Größe und Ladung der Ionen bestimmt, wobei die Wechselwirkung der kugelförmigen Ionen in guter Näherung durch das Coulomb-Gesetz beschrieben werden kann.

\subsubsection{Kovalente Bindung}

Viele charakteristische chemische und physikalische Eigenarten von Molekülen (z. B. ausgeprägt unterschiedliche Reaktionsgeschwindigkeiten) benötigen zum Verständnis die Beschreibung durch andere Formen der chemischen Bindung. Dies wird insbesondere bei den Nichtmetallen deutlich; dazu gehört die Chemie der Kohlenstoffverbindungen (Organische Chemie, Biochemie).

Man kann die kovalente Bindung als Folge der bei der Annäherung zweier Atome (Molekül) eintretenden Verringerung der Gesamtenergie des Systems verstehen, wobei die überschüssige Energie durch einen dritten Stoßpartner abgeführt werden muß. Die Bindungsenergie des Moleküls $\mathrm{Cl}_{2}$ beträgt z. B. $242 \mathrm{~kJ} / \mathrm{mol}$. Für die Verbindung zur Physik der Einzelteilchen ist folgende Beziehung wichtig:

$$
100 \mathrm{~kJ} / \mathrm{mol}=1,0364 \mathrm{eV} / \text { Molekül }
$$

Das bedeutet, $\mathrm{daB}$ ein $\mathrm{Cl}_{2}$-Molekül um 2,5 $\mathrm{eV}$ stabiler ist als zwei getrennte Chloratome. Die kovalente Bindung kann verstanden und beschrieben werden durch ein Elektronenpaar, das zwei Atomen gemeinsam angehört.

Einfacher: Jeweils zwei Atome nutzen ein oder mehrere Elektronenpaare gemeinsam, um das angestrebte Elektronenoktett zu erreichen. Die Anzahl der gemeinsamen Elektronenpaare entspricht der Anzahl der kovalenten Bindungen, die von einem Atom ausgehen $\left(\mathrm{CH}_{4}, 4\right)$. Werden zwei Partner in solchen Atombindungen durch zwei oder drei gemeinsame Elektronenpaare verknüpft, entstehen Doppelbindungen bzw. Dreifachbindungen (Wiedergabe der Valenzelektronen durch Punkte bzw. der gemeinsamen Elektronenpaare durch Striche zwischen den Atomen).

$$
\begin{aligned}
& |\overline{\mathrm{Cl}} \cdot+\cdot \underline{\overline{\mathrm{Cl}}}| \rightarrow|\underline{\mathrm{C}}-\overline{\mathrm{C}}| ; \quad|\dot{\mathrm{N}} \cdot+\cdot \dot{\mathrm{N}}| \rightarrow|\mathrm{N} \equiv \mathrm{N}| \\
& \text { Einfachbindung }
\end{aligned}
$$

Die Entwicklung der Quantenmechanik zu Beginn des 20. Jahrhunderts führte auch zu einem neuen Verständnis in der Molekülbildung. Wie bereits in Kapitel 1 ausführlich ausgeführt wurde, kann man nur Aussagen zu den Aufenthaltswahrscheinlichkeiten der Elektronen machen, wie es die Schrödinger-Gleichung vorführt. Lösungen dieser Gleichung (Wellenfunktionen) für Atome oder Ionen bezeichnet man auch als Atomorbitale, deren Quadrate die Wahrscheinlichkeit beschreiben, im zeitlichen Mittel ein Elektron an einem bestimmten Punkt zu finden (vgl. Kap.1). In der stark vereinfachenden graphischen Darstellung erhält man kugel- oder hantelförmige Gebilde mit positiven und negativen Amplituden und Knoten, in denen sich das Vorzeichen der Funktion ändert.

Für die exemplarische mathematische Behandlung dieser Problematik hat sich das Wasserstoffatom (ein Proton, ein Elektron) bewährt. Die Wellenfunktion mit der niedrigsten Energie ist das 1s-Orbital (Hauptquantenzahl 1). Dieses Orbital ist kugel- 


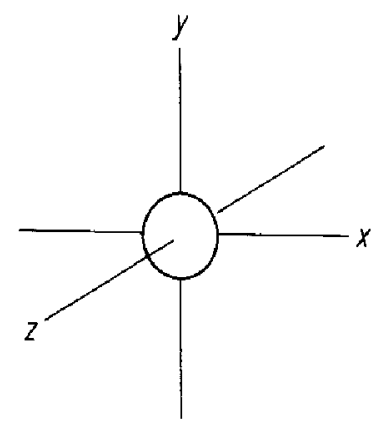

(a)

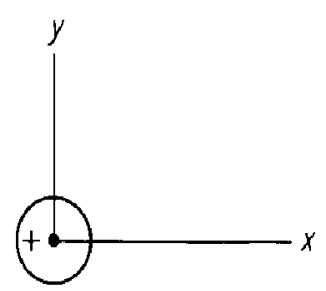

(b)

Abb.4.4 Darstellung eines 1s-Orbitals in dreidimensionaler (a) bzw. zweidimensionaler (b) Form. Das Pluszeichen beschreibt das Vorzeichen der Wellenfunktion.

symmetrisch (Abb. 4.4) und hat keine Knotenebenen. Graphisch stellt man es als diffuse Wolke oder einfach als Kugel bzw. in der Zeichenebene als Kreis dar $(90 \%$ Aufenthaltswahrscheinlichkeit des Elektrons ist innerhalb des Kugelvolumens).

Für das nächsthöhere Energieniveau erhält man als Lösung das ebenfalls kugelsymmetrische 2s-Orbital, das aufgrund des höheren Energiezustands ein größeres Volumen umfaßt.

Im nächsthöheren Fall ergeben sich für das Wasserstoffelektron drei energetisch äquivalente (entartete) Lösungen, bei denen die zylindersymmetrischen, hantelförmigen 2p-Orbitale (siehe Abb. 4.5) bezüglich eines kartesischen Koordinatensystems drei charakteristische räumliche Orientierungen einnehmen können (Knotenebene steht senkrecht auf der Orbitalachse).

Bei der mathematischen Behandlung der Wellengleichung für Atome mit mehr als einem Elektron wird eine genaue Beschreibung sehr schnell außerordentlich aufwen-

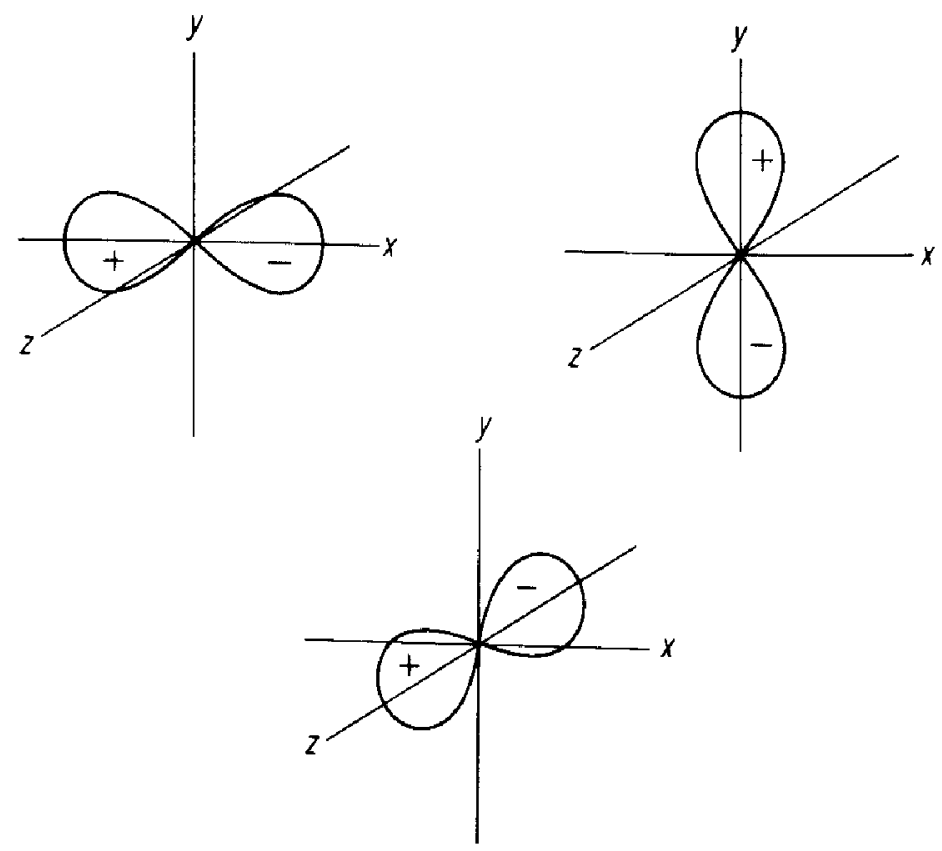

Abb.4.5 Zweidimensionale Darstellung von 2p-Orbitalen. 
dig, da die Wechselwirkungen aller Elektronen untereinander berücksichtigt werden müßten. Erfreulicherweise sind die Form und Knotenebenen der Atomorbitale aller Atome denen des Wasserstoffatoms ähnlich. Die steigende Kernladung läßt jedoch die Größe der Orbitale innerhalb einer Periode nach rechts abnehmen (die besetzten Niveaus mit der kleinsten Ionisationsenergie sind maßgebend für die chemische Bindung).

Mittels eines Energieniveauschemas können wir nun die Elektronen eines jeden Elements auf die Atomorbitale verteilen (Abb. 4.6). Bei diesem Aufbauprinzip müssen einige Regeln beachtet werden (Pauli-Prinzip, Hundsche Regel etc.). Das Ergebnis führt dann zu Elektronenkonfigurationen, welche beispielsweise erkennen lassen, warum Elektronenoktetts besonders stabile Konfigurationen darstellen.

Kovalente Bindungen können besonders anschaulich durch die Überlappung von Atomorbitalen dargestellt werden.

Der wiederum einfachste Fall ist die Bindung zwischen zwei Wasserstoffatomen im $\mathrm{H}_{2}$ (Abb. 4.7). Wir hatten gelernt, daß Atomorbitale Lösungen der Wellengleichung

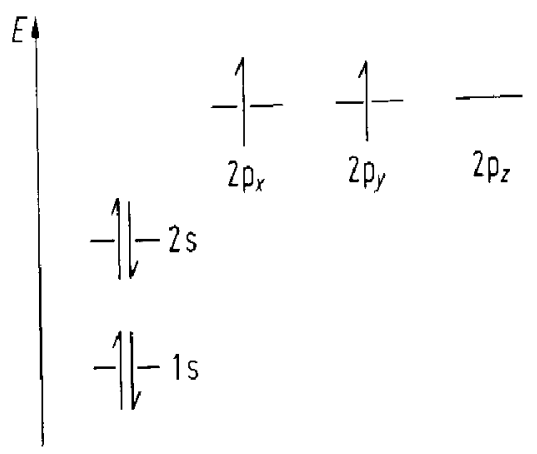

Abb. 4.6 Stabilste Elektronenkonfiguration von atomarem Kohlenstoff. $(1 s)^{2}(2 s)^{2}(2 p)^{2}$. Die jeweils einfache Besetzung zweier p-Orbitale entspricht der Hundschen Regel, die gepaarten Spins im aufgefüllten 1s- und 2s-Orbital sind in Übereinstimmung mit dem Pauli-Prinzip und der Hundschen Regel.
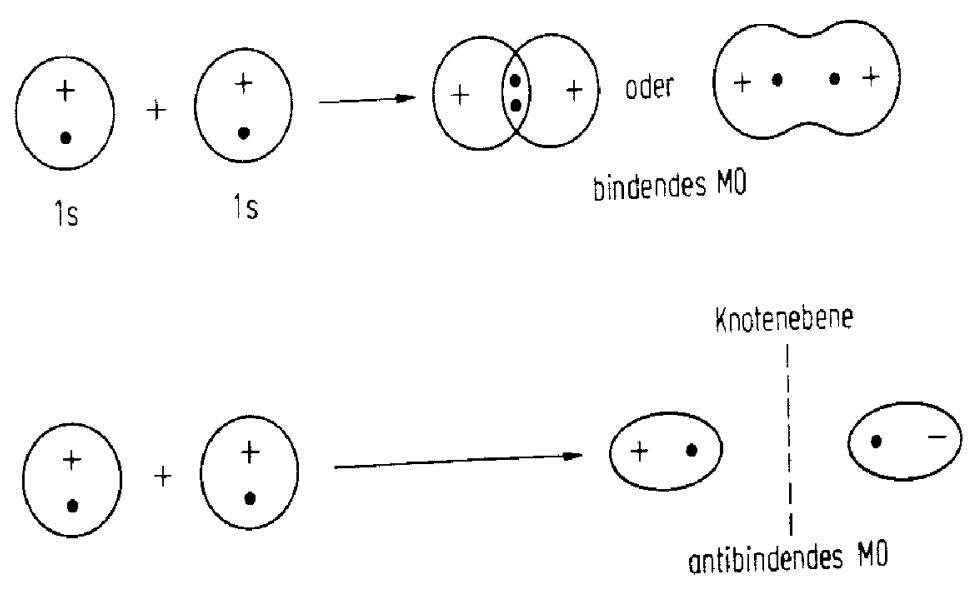

Abb.4.7 Bindende und antibindende Kombination der Wasserstoff-Atomorbitale zu Wasserstoff-Molekülorbitalen. 
im Zentralpotential sind. Wie bei Wellen findet bei der Überlagerung in Bereichen der Wellenfunktion mit gleichem Vorzeichen eine Verstärkung statt. In dieser bindenden Kombination ist die Elektronendichte zwischen den Kernen hoch und führt zur kovalenten Bindung. Die Überlappung der beteiligten Orbitale mit gleichem Vorzeichen führt zum bindenden Molekülorbital, während im antibindenden Molekülorbital (ungleiche Vorzeichen, destabilisierende Wechselwirkung) dort eine Knotenebene erzeugt wird, in der die Amplituden der Wellenfunktion sich zu Null addieren.

Die Überlagerung der beiden 1s-Orbitale zweier Wasserstoffatome ergibt demnach zwei Molekülorbitale (MO). Da das System insgesamt nur zwei Elektronen enthält, besetzen beide das bindende MO mit der niedrigeren Energie (maximal 2 Elektronen pro Orbital mit antiparallelem Spin), was gegenüber den isolierten $\mathrm{H}$-Atomen einen deutlichen Energiegewinn ergibt (Abb. 4.8). So kann man leicht verstehen, warum Wasserstoff als $\mathrm{H}_{2}$-Molekül(e) auftritt, Helium jedoch in atomarer Form. Zwei HeAtome müßten nämlich mit ihren insgesamt vier Elektronen im analogen Energieniveauschema sowohl das bindende als auch das antibindende $\mathrm{MO}$ vollständig auffüllen, was energetisch nicht günstig ist, da der Energieaufwand zur Bildung des antibindenden MO's den Gewinn des bindenden Orbitals weit übertrifft (siehe Korrelationsdiagramme in Abschn. 5.4.3.2).

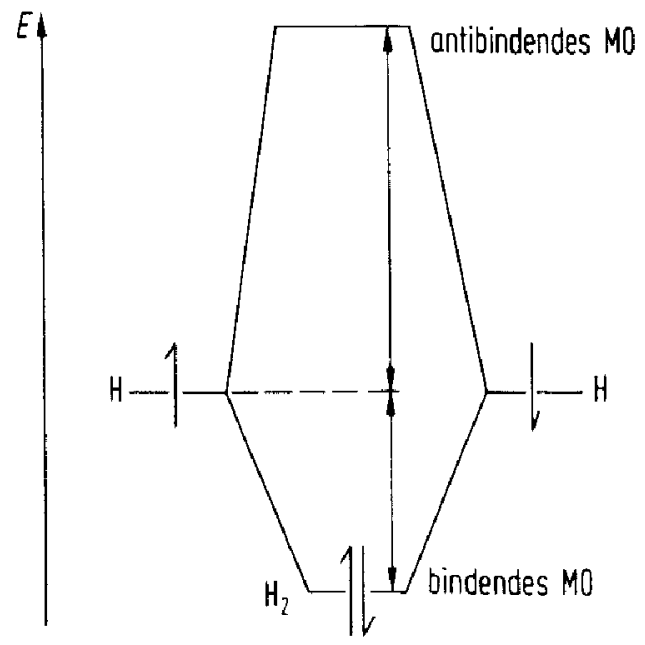

Abb. 4.8 Schematische Darstellung der Kombination der beiden einfach besetzten Wasserstoff-Atomorbitale zu zwei Molekül-Orbitalen.

Die eben beschriebene Aufspaltung von Energieniveaus bei der Kombination von Atomorbitalen läßt sich auf alle Orbitale anwenden. Die Absenkung der Energie des bindenden Molekülorbitals ist hierbei von vielerlei Faktoren abhängig. Beispielsweise spielen geometrische (sterische) Faktoren eine gewichtige Rolle. Dies erkennt man sogleich bei der Einbeziehung der p-Orbitale, die ja nicht kugelsymmetrisch sind. Grundsätzlich sind mehrere Typen von Bindungen möglich. Befindet sich der maximale Ladungszuwachs der Elektronendichte relativ zu den Atomen zylindersymmetrisch auf der Verbindungsachse $z$ wischen den Kernen, so spricht man von einer $\sigma$ Bindung, befindet er sich ober- und unterhalb dieser Achse (wie Abb. $4.9 \mathrm{~d}$ zeigt), so handelt es sich um eine $\pi$-Bindung (Abb. 4.9). Alle Kohlenstoff-Kohlenstoff-Einfach- 


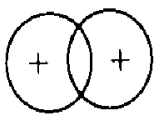

is $1 \mathrm{~s}$

(0)

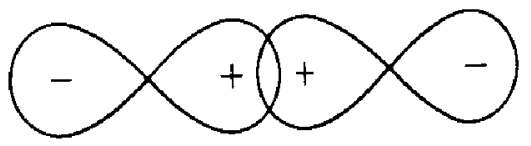

$2 p$

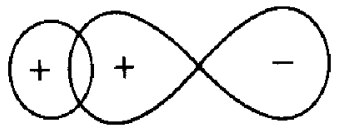

is $2 \mathrm{p}$

(b)

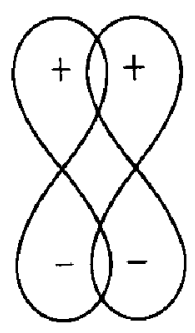

$2 p \quad 2 p$

(d)

(c)

Abb. 4.9 Bindung zwischen Atomorbitalen: (a) 1s und 1s; (b) 1s und 2p; (c) 2p und 2p (entlang der Kernverbindungsachse, $\sigma$-Bindung; (d) $2 p$ und $2 p$ (senkrecht auf der Kernverbindungsachse, $\pi$-Bindung).

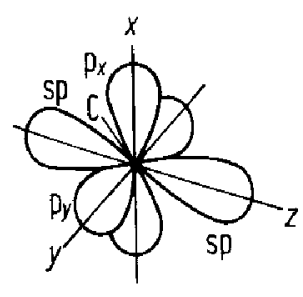

(a)
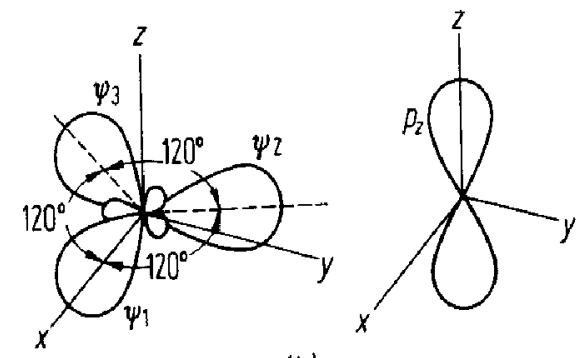

(b)

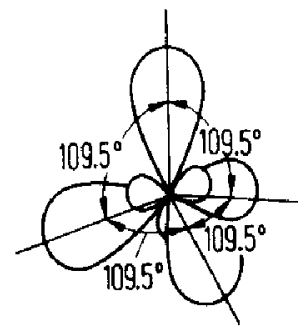

(c)

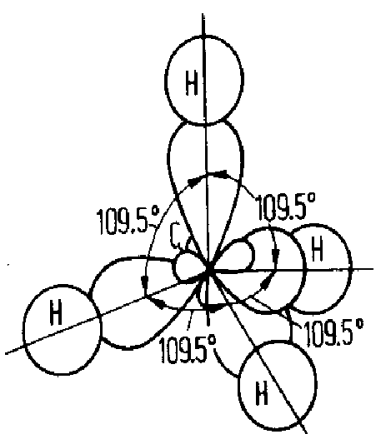

(d)

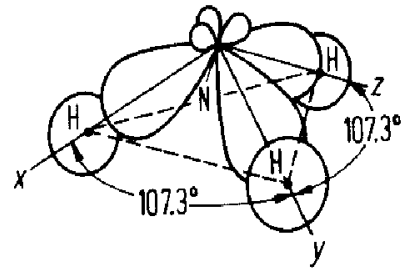

(e)

Abb. 4.10 Wellenfunktionen bei (a) $\mathrm{sp}$-Hybridisierung, (b) $\mathrm{sp}^{2}$-Hybridisierung und (c) $\mathrm{sp}^{3}$ Hybridisierung. (d) Die Hybridisierung im Kohlenstoffatom zu vier $\mathrm{sp}^{3}$-Hybridorbitalen führt zur tetraedrischen Geometrie des Methanmoleküls $\left(\mathrm{CH}_{4}\right)$. (e) Bindungsverhältnisse im Ammoniakmolekül $\mathrm{NH}_{3}$. 
bindungen sind $\sigma$-Bindungen, ihre Mehrfachbindungen enthalten immer zusätzlich $\pi$-Bindungsanteile.

Bei Molekülen aus mehr als zwei Atomen reichen diese Beschreibungen nicht mehr aus. Um die Bindung und auch die Geometrie größerer Moleküle richtig beschreiben zu können, muß man durch eine sinnvolle mathematische Kombination von atomaren Orbitalen neue Hybridorbitale bilden. So entstehen beispielsweise durch geeignete Mischung eines s- und eines p-Orbitals zwei lineare sp-Hybride. Ein s- und zwei pOrbitale liefern drei trigonale $\mathrm{sp}^{2}$-Hybridorbitale und ein s- und drei p-Orbitale vier tetraedrisch angeordnete $\mathrm{sp}^{3}$-Orbitale (Abb. 4.10).

Die Kombination von atomaren Orbitalen höherer Symmetrien zu Bindungsniveaus sind sehr komplex und werden von der Gruppentheorie dominiert. Dem Leser wird das Studium des Abschn. 5.4.3 und der ausführlichen Literatur dieses Spezialgebiets empfohlen.

Diese aufgrund quantenmechanischer Erkenntnisse vertieften Erfahrungen liefern wesentliche Grundlagen für das Verständnis der strukturellen Eigenarten aller Moleküle und eröffneten die Aera der modernen Chemie. Betrachtungen dieser Art erlauben Vorhersagen von Bindungsenergien und -längen, der Molekülsymmetrie und anderer wichtiger Eigenschaften. So ergibt sich beispielsweise die tetraedrische Koordination der C-Atome in gesättigten Kohlenstoffverbindungen mit allen ihren stereochemischen Konsequenzen (vgl. Abschn. 4.3.2).

\subsection{Reaktionsdynamik}

\subsubsection{Chemisches Gleichgewicht}

Was reagiert miteinander und wann? Dies ist eine der grundsätzlichen Fragen an die physikalische Chemie. Die Antwort ist im Einzelfall meist nicht leicht zu verstehen, wenngleich aus Erfahrung, gepaart mit dem Wissen um die Prinzipien der Thermodynamik - also das, was wir in diesem Kapitel kurz vermitteln wollen - in der Regel korrekte Voraussagen möglich sind. Es stellt sich die Frage nach der Spontanität einer Reaktion, d.h. nach ihrer Neigung, von selbst abzulaufen, wobei wir die Geschwindigkeit zunächst unberücksichtigt lassen wollen.

Die Entwicklung von Computerprogrammen auf der Grundlage umfassender Datenbanken wird z. Zt. erfolgreich betrieben und hilft in einigen chemischen Großbetrieben in Einzelfällen bereits bei der Entwicklung von optimalen Reaktionswegen zu einem gewünschten Endprodukt (Möglichkeit zur Minimierung von Nebenprodukten etc.; Umweltschutz).

Beispiel: Die Erzeugung von chemisch interessanten Verbindungen, ausgehend von Stickstoff $\mathrm{N}_{2}$ und Sauerstoff $\mathrm{O}_{2}$ (Luft!), ist speziell aus industrieller Sicht von großem Interesse. In Gegenwart eines Katalysators (vgl. Abschnitt 4.2.2) oder bei hohen Temperaturen ist folgende Reaktion möglich:

$$
\mathrm{N}_{2}+\mathrm{O}_{2} \underset{\mathrm{k}_{2}}{\stackrel{\mathrm{k}_{1}}{\rightleftarrows}} 2 \mathrm{NO}+\Delta \mathrm{G}
$$

Bei genügend hohen Temperaturen werden sich zu Beginn relativ rasch NO-Molekü- 
le bilden, da die Anzahl bzw. die Konzentration der benötigten Reaktionspartner $\mathrm{N}_{2}$ und $\mathrm{O}_{2}$ noch hoch ist. Die Reaktionsrate $\mathrm{R}$ ist gegeben durch $\mathrm{R}_{1}=\mathrm{k}_{1} \mathrm{c}\left(\mathrm{N}_{2}\right) \mathrm{c}\left(\mathrm{O}_{2}\right)$; $c_{A}$ oder $c(A)$ ist die Stoffmengenkonzentration oder einfach Konzentration eines Stoffes A (in der Chemie ist hierfür international noch weitgehend die Abkürzung [A] üblich). Der Verbrauch an Edukten (Ausgangsstoffen, die in die Reaktion eingesetzt werden) wird diese Hinreaktion jedoch verlangsamen. Andererseits können bereits gebildete NO-Moleküle wieder in die Ausgangsstoffe zerfallen, d.h. die sogenannte Rückreaktion mit der Reaktionsrate $R_{2}$ ist ebenfalls möglich $\left(R_{2}=k_{2}\right.$ $\mathrm{c}^{2}$ (NO)). Je mehr NO vorhanden ist, desto ausgeprägter wird diese Rückreaktion sein. Irgendwann wird schließlich ein Punkt erreicht werden, bei dem die Raten der Hin- und Rückreaktion gleich sind $\left(\mathrm{R}_{1}=\mathrm{R}_{2}\right)$.

Dies ist die Bedingung für das chemische Gleichgewicht. Die makroskopische $\mathrm{Zu}$ sammensetzung der Reaktionsmischung hat damit einen dynamischen Gleichgewichtszustand erreicht, d.h. die Hin- und Rückreaktion laufen weiterhin ab, die relativen Konzentrationen der beteiligten Reaktionspartner bleiben jedoch im Mittel konstant. Für die Beschreibung dieses Gleichgewichts hat sich folgende Schreibweise als außerordentlich nützlich erwiesen:

$$
\frac{\mathrm{c}^{2}(\mathrm{NO})}{\mathrm{c}\left(\mathrm{N}_{2}\right) \mathrm{c}\left(\mathrm{O}_{2}\right)}=\frac{\mathrm{k}_{1}}{\mathrm{k}_{2}}=\mathrm{K}
$$

Dieser Ausdruck charakterisiert die Gleichgewichtskonstante $\mathrm{K}$, die das Verhältnis der Konzentrationen der Produkte zu dem der Edukte wiedergibt und als Massenwirkungsgesetz bezeichnet wird.

$$
\mathrm{A}+\mathrm{B} \rightleftarrows \mathrm{C}+\mathrm{D} ; \quad \mathrm{K}=\frac{\mathrm{c}(\mathrm{C}) \mathrm{c}(\mathrm{D})}{\mathrm{c}(\mathrm{A}) \mathrm{c}(\mathrm{B})}
$$

Edukte Produkte

Spontane Reaktionen sind in ihrer Mehrzahl exotherm, d. h. es wird Wärme freigesetzt $(\Delta G<0)$. Es gibt jedoch auch spontan ablaufende endotherme Reaktionen Dieses Phänomen berücksichtigt die freie Enthalpie (Gibb's-Funktion) $G$ :

$$
\mathrm{G}=\mathrm{H}-\mathrm{TS}
$$

Bei jeder spontanen Reaktion unter konstantem Druck und bei konstanter Temperatur muß die freie Enthalpie $G$ unabhängig von der Wärmetönung einer Reaktion stets abnehmen. Im Gleichgewicht ist $\Delta \mathrm{G}=\mathbf{0}$.

Die freie Enthalpie $G$ ist durch zwei Zustandsfunktionen definiert, sie haben folgende Interpretationen:

Die Enthalpie $\mathbf{H}$ ist ein Maß für die Reaktionswärme bei konstantem Druck; die Entropie S (vgl. Bd. I, Abschn. 108/109) eines Systems von chemischen Stoffen berücksichtigt z. B. die Zunahme an Ordnung beim Kristallisieren bzw. die Zunahme an Unordnung beim Auflösen eines Kristalls.

Die Kenntnis der Gleichgewichtskonstanten $\mathrm{K}$ ermöglicht es vorauszusagen, ob und in welcher Richtung eine Reaktion ablaufen wird und (!) wie groß die Anteile der Reaktionspartner sein werden. Die Lage des Gleichgewichts ist unabhängig von der Verwendung eines Katalysators, aber abhängig von Druck und Temperatur. Diese Tatsache ist von außerordentlicher praktischer Bedeutung und wird in Industrie und 
Labor intensiv genutzt, wenn es darum geht, eine Reaktion zur Optimierung der Ausbeute möglichst vollständig in einer Richtung ablaufen zu lassen. Gelingt es darüber hinaus, Anteile des Produkts durch Ausfällen (Löslichkeit), Abdestillieren o.ä. dem Gleichgewicht zu entziehen, kann das System ständig in einem Ungleichgewicht gehalten werden und sogar bei ungünstigen Gleichgewichtskonstanten $\mathrm{K}$ eine praktisch quantitative Umsetzung in der gewünschten Richtung erfolgen.

Beispiel: Bei der Umsetzung der Feststoffe Calciumoxid (gebrannter Kalk) und Kohlenstoff in einem elektrischen Ofen wird bei $2000-3000^{\circ} \mathrm{C}$ Calciumcarbid und Kohlenmonoxid gebildet.

$$
\mathrm{CaO}+3 \mathrm{C} \rightarrow \mathrm{CaC}_{2}+\mathrm{CO} \uparrow
$$

Durch kontinuierliches Entfernen des CO-Gases wird die Reaktion letztlich vollständig in Richtung auf die Calciumcarbidbildung gedrängt.

Spontan ablaufende Reaktionen (fern vom Gleichgewicht) sind oft irreversibel. Gleichgewichtsreaktionen sind reversibel.

\subsubsection{Kinetik, Katalyse}

Das Wissen um die Lage eines Gleichgewichts bzw. die Kenntnis der thermodynamischen Stabilität der beteiligten Reaktionspartner sagt zunächst noch nichts darüber aus, wie schnell sich dieses Gleichgewicht einstellen wird.

Reaktionen zwischen Ionen in Lösung ohne Änderung der Oxidationsstufe (Abschn. 4.2.4) sind außerordentlich schnell. Beispielsweise wird die Geschwindigkeit der Neutralisation einer Säure mit einer Base (Abschn.4.2.3) praktisch ausschließlich durch die Zeit begrenzt, die zur Durchmischung beider Lösungen benötigt wird. (Hier führt nahezu jeder Stoß zwischen den Protonen und den Hydroxid-Ionen zur Bildung von Reaktionswasser; die Reaktion ist diffusionskontrolliert.)

Die Ausbildung eines Niederschlags, z. B. beim Ausfällen von Silberchlorid, erfordert hingegen einige Sekunden.

$$
\mathrm{Ag}^{+}+\mathrm{Cl}^{-} \rightarrow \mathrm{AgCl} \downarrow
$$

Relativ langsam verlaufen auch einige Redoxreaktionen (Abschn. 4.2.4), da nicht jeder StoB zum Übertragen von Elektronen führt.

$$
2 \mathrm{Fe}^{3+}+\mathrm{Sn}^{2+} \rightarrow 2 \mathrm{Fe}^{2+}+\mathrm{Sn}^{4+}
$$

Eine bei Raumtemperatur extrem langsame Reaktion ist hingegen die unter anderen Bedingungen explosionsartig verlaufende Knallgasreaktion.

$$
2 \mathrm{H}_{2}+\mathrm{O}_{2} \rightarrow 2 \mathrm{H}_{2} \mathrm{O}
$$

Die Reaktionsgeschwindigkeit hängt eben nicht nur von der Zusammensetzung der reagierenden Substanzen ab, sondern auch von deren physikalischem Zustand, von der Güte der Vermischung und von Druck, Temperatur und Konzentration. Entscheidend können auch besondere physikalische Bedingungen wie Bestrahlung mit Licht bestimmter Wellenlänge oder die Gegenwart anderer Substanzen (Katalysatoren) sein, die die Reaktionen beeinflussen, sich dabei aber selbst nicht verändern. 
Hier hilft das Wissen um die Prinzipien der chemischen Kinetik. Die Reaktionsrate wird um so größer sein, je höher die Wahrscheinlichkeit eines Zusammenstoßes der Partner ist. Das bedeutet, daß größere Teilchenzahl pro Volumen (Konzentration) und höhere Temperaturen im allgemeinen den Umsatz erhöhen, wobei die Reaktionswahrscheinlichkeit über alle räumlichen Orientierungen der Stoßpartner gemittelt wird. Man unterscheidet zwischen Reaktionen 1. und höherer Ordnung, je nachdem, ob die Reaktionsgeschwindigkeit nur von der Konzentration eines Reaktionspartners $\left(\mathrm{c}_{\mathrm{A}}\right)$ abhängt, oder ob die Konzentrationen mehrerer bzw. aller beteiligten Reaktionspartner $\left(c_{A}, c_{B}, c_{C} \ldots\right)$ entscheidend sind.

Zeit-Gesetz für Reaktionen 1.Ordnung (z. B. radioaktiver Zerfall):

$$
\frac{\mathrm{dc}_{\mathrm{A}}}{\mathrm{dt}}=-\mathrm{kc} \mathrm{c}_{\mathrm{A}} \text { und } \mathrm{c}_{\mathrm{A}}=\mathrm{c}_{\mathrm{o}} \mathrm{e}^{-\mathrm{kt}}
$$

Aber nicht jeder Zusammenstoß zwischen den Reaktionspartnern führt zur Reaktion, d.h. nicht alle Teilchen einer Substanz sind jederzeit reaktionsbereit. Es reagieren nur solche miteinander, deren Gesamtenergie einen gewissen Minimalbetrag überschreitet. Diese Mindestenergie zur Überwindung der vorliegenden Potentialschwellen wird als Arrheniussche Aktivierungsenergie bezeichnet und bestimmt letztlich die tatsächliche Reaktionsgeschwindigkeit (Abb. 4.11) (die freie Enthalpie $\mathrm{G}$ ist nur ein Maß für die „Triebkraft" einer Reaktion).

Ein Katalysator wirkt sich nicht auf die freie Enthalpie aus, kann aber die Aktivierungsbarriere durch einen veränderten (modifizierten) Reaktionsmechanismus entscheidend senken und die Reaktionen damit erheblich beschleunigen.

Wird die katalytische Wirkung dadurch hervorgerufen, da $B$ die Reaktionspartner an die Oberfläche einer festen Phase gebunden sind, spricht man von heterogener Katalyse (vgl. Bd. VI, Kap. 3). Sie spielt bei vielen technischen Prozessen eine entscheidende Rolle und ist im Zusammenhang mit der Entgiftung von Abgasen (z. B. „Katalysator-Auto") allgemein bekannt geworden. Dort werden Edelmetallpartikel z. B. aus Platin oder Palladium verwendet, die in keramische Oberflächen eingebettet sind.

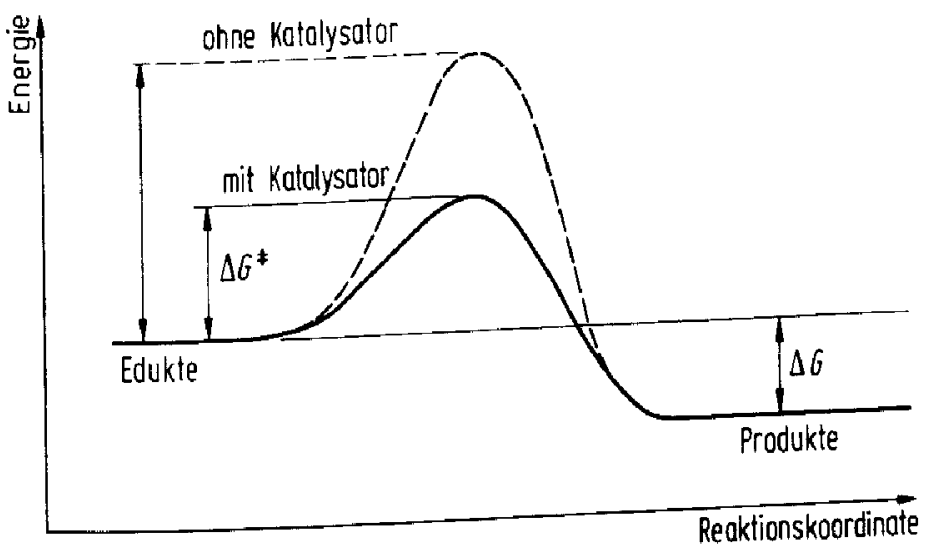

Abb.4.11 Ein Katalysator beschleunigt eine ohnehin spontane Reaktion, indem er einen alternativen Reaktionsweg mit niedrigerer Aktivierungsenergie $\Delta G^{\ddagger}$ ermöglicht. 
Preisgünstige Verfahren, die zugleich Umweltbelastungen reduzieren helfen, sind häufig abhängig von der Entwicklung und dem Einsatz geeigneter (in der Regel heterogener) Katalysatoren (Tab. 4.2). Sie ermöglichen, bei möglichst niedrigen Temperaturen und Drücken (Herabsetzen der Aktivierungsenergie; Energieeinsparung) und mit leicht verfügbaren Reagenzien (z. B. Luftsauerstoff statt teurer und möglicherweise schwer zu entsorgender Oxidationsmittel) arbeiten zu können. Im Labormaßstab hingegen lohnt sich für kleine Mengen häufig keine aufwendige Katalysatorsuche bzw. -entwicklung.

Tab.4.2 Beispiele für den Einsatz heterogener Katalysatoren bei wichtigen industriellen Verfahren.

\begin{tabular}{|c|c|}
\hline Verfahren & Katalysator \\
\hline Ammoniaksynthese (Haber-Bosch-Verfahren) & $\mathrm{Fe} / \mathrm{Al}_{2} \mathrm{O}_{3} / \mathrm{K}_{2} \mathrm{O}$ \\
\hline $\begin{array}{l}\text { Oxidation von Schwefeldioxid zu Schwefelsăure (Kontakt- } \\
\text { verfahren) }\end{array}$ & $\mathrm{V}_{2} \mathrm{O}_{5} / \mathrm{SiO}_{2}$ \\
\hline $\begin{array}{l}\text { Oxidation von Ammoniak zu Stickoxiden zur Herstellung } \\
\text { von Salpetersäure }\end{array}$ & $\mathrm{Pt} / \mathrm{Rh}$ \\
\hline Fischer-Tropsch-Synthese & $\mathrm{Fe}, \mathrm{Co} / \mathrm{ThO}_{2} / \mathrm{MgO}$ \\
\hline \multicolumn{2}{|l|}{ Synthese von Methanol: } \\
\hline - Hochdruckverfahren & $\mathrm{ZnO} / \mathrm{Cr}_{2} \mathrm{O}_{3}$ \\
\hline - Niederdruckverfahren & $\mathrm{CuO} / \mathrm{Cr}_{2} \mathrm{O}_{3}$ \\
\hline \multicolumn{2}{|l|}{ Konvertierung von Kohlenmonoxid: } \\
\hline - Hochtemperaturkonvertierung & $\mathrm{Fe}_{2} \mathrm{O}_{3} / \mathrm{Cr}_{2} \mathrm{O}_{3}$ \\
\hline - Tieftemperaturkonvertierung & $\mathrm{ZnO} / \mathrm{CuO} / \mathrm{Cr}_{2} \mathrm{O}_{3}$ \\
\hline Dampfreformieren von Kohlenwasserstoffen & $\mathrm{Ni} / \mathrm{CaCO}_{3}$ \\
\hline Katalytisches Cracken von Kohlenwasserstoffen & $\mathrm{Al}_{2} \mathrm{O}_{3} / \mathrm{SiO}_{2}$ (Zeolithe) \\
\hline Hydrospalten von Kohlenwasserstoffen & $\mathrm{Ni} / \mathrm{Pd} / \mathrm{Al}_{2} \mathrm{O}_{3} / \mathrm{SiO}_{2}$ (Zeolithe) \\
\hline Reformieren von Schwerbenzin & $\mathrm{Pt} / \gamma-\mathrm{Al}_{2} \mathrm{O}_{3}$ \\
\hline Isomerisierung der $\mathrm{C}_{8}$-Aromaten & $\mathrm{Pt} / \mathrm{Al}_{2} \mathrm{O}_{3} / \mathrm{SiO}_{2}$ (Zeolith) \\
\hline Hydroraffination & $\begin{array}{l}\mathrm{NiS} / \mathrm{WS}_{2} / \mathrm{Al}_{2} \mathrm{O}_{3} \text { oder } \\
\mathrm{CoS} / \mathrm{MoS}_{2} / \mathrm{Al}_{2} \mathrm{O}_{3}\end{array}$ \\
\hline Selektivhydrierung von Phenol zu Cyclohexanon & $\mathrm{Pd} / \mathrm{Al}_{2} \mathrm{O}_{3}$ \\
\hline Dehydrierung von Ethylbenzol zu Styrol & $\mathrm{Fe}_{3} \mathrm{O}_{4} / \mathrm{Cr}_{2} \mathrm{O}_{3} / \mathrm{K}_{2} \mathrm{O}$ \\
\hline Oxidation von Ethyler a Ethylenoxid & $\mathrm{Ag} / \alpha-\mathrm{Al}_{2} \mathrm{O}_{3}$ \\
\hline $\begin{array}{l}\text { Oxidation von Benzol zu Maleinsäureanhydrid sowie von } \\
\text { o-Xylol zu Phthalsäureanhydrid }\end{array}$ & $\mathrm{V}_{2} \mathrm{O}_{5} / \mathrm{SiO}_{2}$ \\
\hline Abgasenachverbrennung (Autokatalysator) & Pt oder $\mathrm{Pd}$ auf $\mathrm{Al}_{2} \mathrm{O}_{3}$ \\
\hline
\end{tabular}

\subsubsection{Säure-Base-Reaktionen}

Das Wort Säure ist sicher nicht erst seit der Diskussion um den sauren Regen der im allgemeinen Sprachgebrauch am weitesten verbreitete Begriff aus dem Bereich der Chemie. Erste Assoziation sind der saure Geschmack, die Magensäure und das ätzende (,zerstörende") bzw. korrosionsfördernde Verhalten.

Der Begriff Säure umfaßt so viel, da $B$ es (auch historisch bedingt) verschiedene 
Definitionen gibt. Für die meisten Fälle ist folgende Beschreibung ausreichend: Eine Säure ist eine Substanz, die beim Auflösen in Wasser in einfach positiv geladene Wasserstoffionen $\left(\mathrm{H}^{+}\right)$und ein entsprechendes Anion $\left(\mathrm{A}^{-}\right)$zerfällt (dissoziiert) und somit einen Protonendonator darstellt. Das Gegenstück ist eine Base, die ganz analog in ein Kation $\left(\mathrm{B}^{+}\right)$und einfach negativ geladene Hydroxid-Ionen $\left(\mathrm{OH}^{-}\right)$zerfällt bzw. über $\mathrm{OH}^{-}+\mathrm{H}^{+} \rightleftarrows \mathrm{H}_{2} \mathrm{O}$ als Protonenakzeptor wirkt.

$$
\begin{aligned}
& \mathrm{HA} \rightleftarrows \mathrm{H}^{+}+\mathrm{A}^{-} \\
& \mathrm{BOH} \rightleftarrows \mathrm{B}^{+}+\mathrm{OH}^{-}
\end{aligned}
$$

Ein wichtiges $\mathrm{Ma} ß$ für die Säurestärke ist der Dissoziationsgrad. Während starke Mineralsäure wie Salzsäure $(\mathrm{HCl})$ oder Schwefelsäure $\left(\mathrm{H}_{2} \mathrm{SO}_{4}\right)$ praktisch vollständig dissoziiert sind, ist dies bei einer schwachen Säure wie Essigsäure $\left(\mathrm{CH}_{3} \mathrm{COOH}\right)$ nicht der Fall. Sie liegt auch in wäßriger Lösung zu einem Großteil in der undissoziierten Form vor.

$$
\mathrm{CH}_{3} \mathrm{COOH} \leftrightarrows \mathrm{CH}_{3} \mathrm{COO}^{-}+\mathrm{H}^{+}
$$

Wasser selbst kann als Säure und auch als Base fungieren (amphoteres Verhalten), denn die Eigendissoziation des Wasser liefert folgendes Gleichgewichtssystem:

$$
\mathrm{H}_{2} \mathrm{O} \rightleftarrows \mathrm{H}^{+}+\mathrm{OH}^{-}
$$

Den Dissoziationsgrad kann man z. B. mit Hilfe der elektrischen Leitfähigkeit ermitteln. Er ist temperaturabhängig und bei Wasser sehr klein. Die Konzentration an $\mathrm{H}^{+}$Ionen (Protonen) in reinstem Wasser beträgt bei Raumtemperatur $10^{-7}[\mathrm{~mol} / \mathrm{l}]$. Da je nach Verbindung die Konzentration an $\mathrm{H}^{+}$-Ionen in wäßriger Lösung sehr variieren kann, hat man als praktikablere Einheit die $\mathrm{pH}$-Skala eingeführt, die inzwischen auch allgemeinere Popularität erlangt hat. Der $\mathrm{pH}$-Wert ist definiert als der negative dekadische Logarithmus der Wasserstoffionen-Konzentration.

$$
\mathrm{pH}=-\lg \mathrm{c}_{\mathrm{H}^{+}}
$$

Reines Wasser weist somit einen $\mathrm{pH}-$ Wert von 7 auf, der als neutral eingestuft wird. Bei einem $\mathrm{pH}$-Wert $<7$ reagiert die Lösung sauer, einen $\mathrm{pH}$-Wert $>7$ erhält man bei basischen Lösungen. Speziell biologische Systeme reagieren sehr empfindlich auf Änderungen des $\mathrm{pH}$-Wertes. Einfache Messungen des $\mathrm{pH}$-Wertes gelingen mit Hilfe von Indikatoren (organische Farbstoffe), die bei bestimmten $\mathrm{pH}$-Werten einen charakteristischen Farbumschlag (verbunden mit einer Änderung ihrer chemischen Struktur) zeigen. Es existiert eine Palette preiswerter Indikatoren, die den gesamten Bereich abdecken und mit denen es einfach gelingt, den $\mathrm{pH}$-Wert einer wäßrigen Lösung zu bestimmen (Teststäbchen).

Eine absolut elementare chemische Reaktion ist die Neutralisation einer Säure mit einer Base (Vorsicht! Derartige Reaktionen verlaufen stark exotherm und sollten nur mit verdünnten Lösungen durchgeführt werden). Hier reagieren gemäß dem Gleichgewicht des Wassers $\mathrm{H}^{+}$-Ionen mit $\mathrm{OH}^{-}$-Ionen unter Bildung von Wasser, während die entsprechenden Gegenionen nach dem Abdampfen des Wassers als Salz isoliert werden können. Auf diese Art und Weise erhält man beispielsweise aus Salzsäure $(\mathrm{HCl})$ und Natronlauge $(\mathrm{NaOH})$ schließlich Wasser $\left(\mathrm{H}_{2} \mathrm{O}\right)$ und Kochsalz $(\mathrm{NaCl})$.

$$
\mathrm{HCl}+\mathrm{NaOH} \rightarrow \mathrm{NaCl}+\mathrm{H}_{2} \mathrm{O}
$$




\subsubsection{Redoxverhalten}

Eine Vielzahl chemischer Prozesse findet unter Übertragung von Elektronen von einem Reaktionspartner auf einen anderen statt. Dies kann an einer stationären Elektrode (fest) ablaufen, aber auch in homogener Phase. Direkt hiermit verknüpft sind elementare Experimente der Physik und der Chemie, mit denen Begriffe wie Elektrolyse (Bd. V, Kap. 5), Spannungsreihe (Bd. VI, Kap. 5) etc. vermittelt werden. Die chemischen Bausteine, die hier im Normalfall benötigt werden, müssen demnach in der Lage sein, relativ leicht Elektronen aufzunehmen bzw. abzugeben. Die Elektronegativität ist im PSE im hinteren Einbanddeckel angegeben.

Wir wollen uns zunächst der Elektrolysereaktion zuwenden, bei der unter dem Einfluß einer externen Spannungsquelle mit Hilfe zweier Elektroden Elektronentransporte stattinden: Natriumchlorid $(\mathrm{NaCl})$ schmilzt oberhalb von $801^{\circ} \mathrm{C}$. Taucht man in eine solche Salzschmelze zwei an eine Spannungsquelle angeschlossene Kohleelektroden, so scheidet sich an der Kathode metallisches Natrium ab. Die in der Schmelze vorliegenden $\mathrm{Na}^{+}$-Ionen werden an der Elektrode entladen. Da die Kathode Elektronen liefert, und die Aufnahme von Elektronen als Reduktion bezeichnet wird, werden bei diesem Teilprozeß $\mathrm{Na}^{+}$-Ionen zu Na-Metall reduziert. Die entsprechende Oxidation ist die Abgabe von Elektronen, die zeitgleich an der Anode abläuft. Hier entwickelt sich Chlorgas durch anodische Oxidation von $2 \mathrm{Cl}^{-} \mathrm{zu} \mathrm{Cl}_{2}$. (In wäßriger Lösung beobachtet man bei der Elektrolyse von $\mathrm{NaCl}$ Folgereaktionen der Primärprodukte mit Wasser.) Die Elektrolyse von $\mathrm{H}_{2} \mathrm{O}$ liefert $\mathrm{H}_{2}$ und $\mathrm{O}_{2}$, und wird durch Protonen $\left(\mathrm{H}^{+}\right)$katalysiert.

Im Fall einer wiederaufladbaren Batterie (z. B. Bleiakkumulator) regeneriert die Elektrolysereaktion mit Hilfe einer Spannungsquelle den chemischen Energiespeicher (Ladevorgang). Danach kann die Rückreaktion der Elektrolyse ablaufen. Der im thermodynamischen Sinne spontane Redoxprozeß liefert jetzt selbst Energie und dient bei Anschluß eines Verbrauchers seinerseits als „Elektronenpumpe“. Nach Abschluß dieser spontanen chemischen Reaktion (Entladung) liegen dann erneut die Ausgangsstoffe für die Elektrolyse vor, mit der der Kreislauf fortgesetzt werden kann (Voraussetzungen: reversibler ProzeB, abgeschlossenes System etc.). Wird z. B. im Bleiakkumulator $\mathrm{H}_{2}$ freigesetzt, muß $\mathrm{H}_{2} \mathrm{O}$ nachgefüllt werden.

Das chemische Potential (Gleichgewichtskriterium für die Betrachtung thermodynamischer Prozesse) einer spontanen Reaktion wird in der Praxis intensiv für gewünschte Stoffumwandlungen genutzt. So ist metallisches Natrium ein effizientes Reduktionsmittel, d.h. es gibt Elektronen ab und wird selbst im Sinne der Teilreaktion $\mathrm{Na} \rightarrow \mathrm{Na}^{+}+\mathrm{e}^{-}$oxidiert. Der Partner kann z. B. Wasser sein, das bei gleichzeitiger Bildung von $\mathrm{OH}^{-}$-Ionen $\mathrm{zu} \mathrm{H}_{2}$-Gas reduziert wird (Vorsicht! Die Reaktion läuft fast explosiv ab). Ein sehr preiswertes Reduktionsmittel steht mit der Kohle zur Verfügung. Elementarer Kohlenstoff reduziert beispielsweise Eisenoxide (Eisenerz) bei hohen Temperaturen unter Abgabe von Elektronen zu metallischem Eisen und wird selbst zu $\mathrm{CO}_{2}$ oxidiert. Bekannte Oxidationsmittel sind der Luftsauerstoff und das Permanganat-Ion $\mathrm{MnO}_{4}^{-}$.

Diese Betrachtungsweise ist von der Praxis geprägt und darf nicht absolut gesehen werden. Bei der Analyse der Spannungsreihe erkennt man nämlich sofort, daß es die elektrochemischen Standardpotentiale sind, die den Reaktionsverlauf festlegen. Die Frage, ob es sich bei einem bestimmten Stoff um ein Reduktions- oder Oxidations- 
mittel handelt, wird demnach vom jeweiligen Reaktionspartner bestimmt: Während Kupfer-Ionen $\left(\mathrm{Cu}^{2+}\right)$ gegenüber Zink ( $\left.\mathrm{Zn}\right)$ als Oxidationsmittel auftreten, wird $\mathrm{Cu}$ durch Silber-Ionen $\mathrm{Ag}^{+}$oxidiert. Der allgegenwärtige Prozeß der Korrosion soll als wichtiger, wenn auch in der Regel unerwünschter Redoxvorgang aus der Praxis genannt werden. Es bilden sich durch Berühren zweier unterschiedlich edler Metalle (Spannungsreihe) sogenannte Lokalelemente, die in Gegenwart von Wasser $\left(\mathrm{H}_{2} \mathrm{O}\right.$; Luftfeuchtigkeit) zur $\mathrm{H}_{2}$-Bildung (Reduktionsschritt) und „Auflösen“" des unedleren Metalls durch Oxidation zum Kation führen (Salz- bzw. Oxidbildung; , Rosten“ von Eisen ergibt hydratisierte Eisenoxide).

$$
2 \mathrm{Fe} \stackrel{\mathrm{H}_{2} \mathrm{O}(\mathrm{Cu})}{\longrightarrow}, 3 \mathrm{H}_{2} \uparrow+\underset{\text {,Rost" }}{\mathrm{Fe}_{2} \mathrm{O}_{3} \cdot \mathrm{nH}_{2} \mathrm{O}}
$$

\subsection{Synthese}

Dieser Abschnitt befaßt sich vornehmlich mit Kohlenstoffverbindungen und beleuchtet beispielhaft neben wenigen grundlegenden auch einige moderne Aspekte der Organischen Chemie.

\subsubsection{Reaktionsmöglichkeiten und Mechanismen}

Obwohl nur relativ wenige Elemente neben Kohlenstoff am Aufbau organischer Produkte beteiligt sind, kennt man doch mit nahezu 4 Millionen gegenüber knapp 100000 erheblich mehr charakterisierte organische als anorganische Verbindungen. Eindrucksvoll katalogisiert sind sie im Beilstein bzw. Gmelin. Einen schnellen und sehr aktuellen Zugriff erlauben die Chemical Abstracts (CA) bzw. die CA-Datenbank und einige weitere Datenbanken.

Die Vielfalt und Sonderstellung der Kohlenstoffverbindungen (pro Jahr kommen ca. 100000 hinzu) ist insbesondere durch die Eigenschaft des Kohlenstoffs begründet, mit sich und anderen Elementen kovalente Einfach- und Mehrfachbindungen zu relativ beständigen Ketten, Ringen und dreidimensionalen Netzen ausbilden zu können (Voraussetzung: besondere Stellung im Periodensystem; IV. Hauptgruppe, 1. Langperiode). Der relativ kleine Atomradius begünstigt eine optimale Überlappung der bindenden Atomorbitale und ermöglicht sehr stabile Bindungen. Eine analoge Siliciumchemie ist wegen des wesentlich größeren Bindungsabstands und der damit deutlich geschwächten Si-Si-Bindung weitaus weniger ausgeprägt.

Wie kann man nun Verbindungen mit einer gewünschten Struktur aufbauen? Es existieren viele empirische und semiempirische Regeln und Rezepte. Im Prinzip ist eine Lösung auf der Basis der Quantenmechanik sogar berechenbar, aber die Vielzahl der zu beachtenden Parameter zwingt zu Vereinfachungen, die bei komplexeren Aufgaben eindeutige Entscheidungen zwischen den unübersehbar vielen Reaktionsmöglichkeiten nicht mehr zulassen. An dieser Stelle ist ein Molekülarchitekt gefragt, der theoretisch und praktisch (präparativ) geschult und mit den wesentlichen analytischen Verfahren vertraut, Reaktionswege entwickeln muB.

Man unterscheidet z. B. zwischen Substitutions-, Additions- und Eliminierungsreak- 
Substitutionsreaktion<smiles>CC(C)(C)C(C)(C)C(C)(C)CC[18CH2]</smiles>

Additionsreaktion

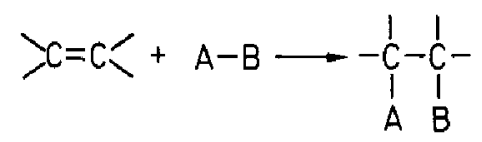

Eliminierungsreaktion<smiles>[B]CCCCCCCCCCCC(C)(C)C([B])(C)C</smiles>

Abb.4.12 Grundlegende Reaktionstypen der Organischen Chemie.

tionen (Abb.4.12). Weiterhin werden zur genaueren Charakterisierung die Begriffe nucleophil (elektronenreich; es werden Positionen mit Elektronenmangel angegriffen), elektrophil (elektronenarm) oder radikalisch hinzugefügt.

Im allgemeinen spielen sogenannte funktionelle Gruppen eine zentrale Rolle. So wird durch Einbau eines Heteroatoms als Folge der Elektronegativitätsunterschiede die C-X-Bindung der kovalenten Bindungspartner polarisiert und ein entsprechendes, weitgehend vorhersagbares Reaktionspotential (nucleophil, elektrophil, radikalisch; Redoxverhalten) erzeugt (Abb. 4.13). Dazu kommen mehrere andere Kriterien, welche den Verlauf elektrocyclischer Reaktionen bestimmen [2].

In der Regel kollidieren bei organischen Reaktionen zwei Moleküle, wobei eine oder mehrere Bindungen gebrochen und entsprechend neue Bindungen gebildet werden. Hierbei werden zumeist eine oder mehrere Zwischenstufen (reaction intermediates) durchlaufen (Abb.4.14).

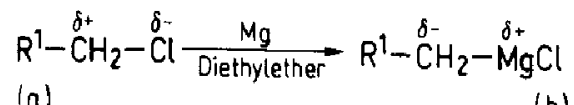

(a)<smiles>[R]CC([R])C([R])=O</smiles>

(d)

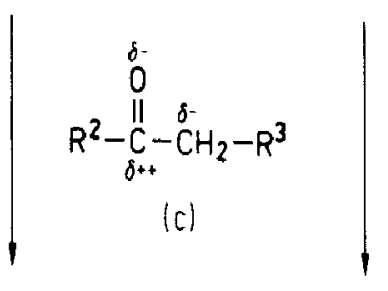<smiles>[R]CC([R])(O)C[R]</smiles>

(e)

Abb. 4.13 Funktionelle Gruppen können eine Polarisierung von Bindungen bewirken und ein entsprechendes Reaktionspotential erzeugen: Das Alkylhalogenid (a) reagiert unter geeigneten Bedingungen mit dem Keton (c) gemäß der angegebenen Polarisierung als Elektrophil zum Calkylierten Produkt (d). Freigesetzte $\mathrm{HCl}$ wird durch Basenzugabe neutralisiert. Die Umpolung der Reaktivität von (a) gelingt durch Umsetzen mit Magnesium zur metallorganischen (Grignard)Verbindung (b). Der jetzt nucleophile Angriff findet am Carbonylkohlenstoff des Ketons (c) statt und liefert nach Hydrolyse den Alkohol (e). 


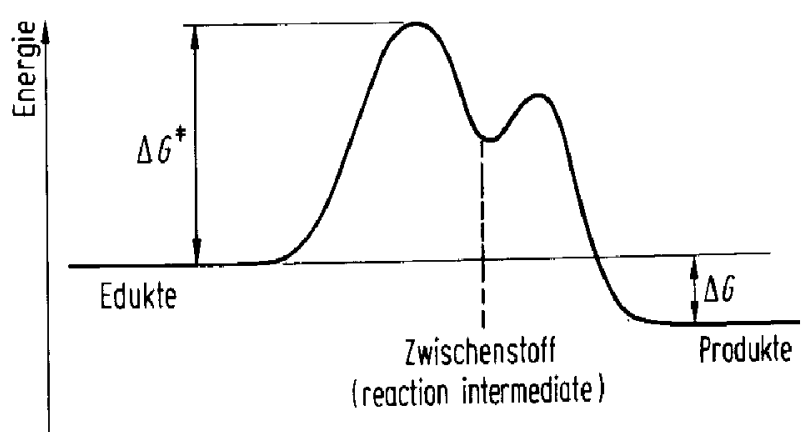

Reaktionskoordinate

Abb.4.14 Energiediagramm einer Reaktion.

Tab.4.3 Wichtige Substanzklassen in der Organischen Chemie.

\begin{tabular}{|c|c|c|c|}
\hline Substanzklasse & $\begin{array}{l}\text { Funktionelle } \\
\text { Gruppe }\end{array}$ & Beispiel & Struktur \\
\hline Alkene & $>\mathrm{C}=\mathrm{C}<$ & Ethen & 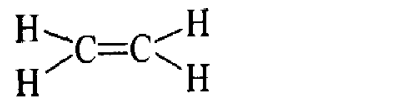 \\
\hline Alkine & $-\mathrm{C} \equiv \mathrm{C}-$ & Acetylen & $\mathrm{H}-\mathrm{C} \equiv \mathrm{C}-\mathrm{H}$ \\
\hline Alkohole & $-\mathrm{OH}$ & Ethanol & $\mathrm{H}_{3} \mathrm{C}-\mathrm{CH}_{2}-\mathrm{OH}$ \\
\hline Halogenide & $-X$ & Methylchlorid & $\mathrm{H}_{3} \mathrm{C}-\mathrm{Cl}$ \\
\hline Amine (prim.) & $-\mathrm{NH}_{2}$ & Methylamin & $\mathrm{H}_{3} \mathrm{C}-\mathrm{NH}_{2}$ \\
\hline $\begin{array}{l}\text { Nitro- } \\
\text { Verbindungen }\end{array}$ & $-\mathrm{NO}_{2}$ & Nitromethan & $\mathrm{H}_{3} \mathrm{C}-\mathrm{NO}_{2}$ \\
\hline Aldehyde & $-\mathrm{C}-\mathrm{H}$ & Acetaldehyd & $\mathrm{H}_{3} \mathrm{C}-\stackrel{\|}{\mathrm{C}}-\mathrm{H}$ \\
\hline Ketone & $-\mathrm{C}-$ & Aceton & $\mathrm{H}_{3} \mathrm{C}-\stackrel{\coprod}{\mathrm{C}}-\mathrm{CH}_{3}$ \\
\hline Carbonsäuren & $-\stackrel{\mathrm{C}}{\mathrm{C}}-\mathrm{OH}$ & Essigsäure & $\mathrm{H}_{3} \mathrm{C}-\stackrel{\coprod}{\mathrm{C}}-\mathrm{OH}$ \\
\hline Ester & $-\stackrel{C}{C}-\mathrm{O}-$ & $\begin{array}{l}\text { Essigsäure- } \\
\text { ethylester }\end{array}$ & $\mathrm{H}_{3} \mathrm{C}-\stackrel{\mathrm{O}}{\mathrm{C}}-\mathrm{O}-\mathrm{CH}_{2}-\mathrm{CH}_{3}$ \\
\hline Amide & $-\mathrm{C}-\mathrm{NH}_{2}$ & $\begin{array}{l}\text { Essigsäure- } \\
\text { amid }\end{array}$ & $\mathrm{H}_{3} \mathrm{C}-\stackrel{\mathrm{C}}{\mathrm{C}}-\mathrm{NH}_{2}$ \\
\hline Nitrile & $-\mathrm{C} \equiv \mathrm{N}$ & Acetonitril & $\mathrm{H}_{3} \mathrm{C}-\mathrm{C} \equiv \mathrm{N}$ \\
\hline
\end{tabular}


Für sämtliche hier beschriebenen Reaktionstypen gelten die in Abschn. 4.2 ausgeführten Gesetzmäßigkeiten der Energetik und des chemischen Gleichgewichts. Kennt man den genauen Reaktionsablauf - die Aufeinanderfolge von Reaktionsschritten, den Bindungsbruch, die neue Bindungsbildung, die Einzelheiten der Elektronenverschiebung, den Zeitverlauf und die Reaktionskinetik - dann kann der Reaktionsmechanismus einer Reaktion angegeben werden. Aufgrund vergleichender Überlegungen werden verwandte Mechanismen und Korrelationen gefunden, für die sich im Laufe der Zeit quasi als Gedächtnisstütze nach dem Erfinder sogenannte Namensreaktionen (Wittig ${ }^{1}$-Reaktion, Michael-Addition etc.) etabliert haben; so wird häufig die große Fülle von experimentellen Erfahrungen und Erkenntnissen in den Naturwissenschaften geordnet.

Eine echte Alternative bietet das Ordnen der oben kurz erwähnten und in der Praxis unglaublich vielfältigen funktionellen Gruppen in verschiedene Substanzklassen (Tab. 4.3; es gibt natürlich auch entsprechende polyfunktionelle Verbindungen). Das Wissen um deren Darstellungsmöglichkeiten und spezifisches Synthesepotential gehört zum absoluten Grundwissen eines präparativ arbeitenden Wissenschaftlers, denn hiermit hat er das notwendige, fein abgestufte Arsenal an Reaktionspartnern zur Verfügung, das er dann gezielt für sein spezielles Problem einsetzen kann, also auch zur Synthese von Makromolekülen. Die Abb. 4.15 zeigt einige ausgewählte, häufig beschrittene Reaktionswege.

Am Beispiel des Benzols soll eine speziell in der Chemie organischer Verbindungen wichtige Struktur-Reaktivitäts-Beziehung verdeutlicht werden. Benzol ist ungewöhnlich reaktionsträge und geht nicht die für Alkene üblichen Additionsreaktionen (Abb. 4.12) ein. Alle sechs Kohlenstoffatome sind $\mathrm{sp}^{2}$-hybridisiert und jedes p-Orbital überlappt gleichmäßig mit seinen beiden Nachbarn. Die auf diese Weise delokali-
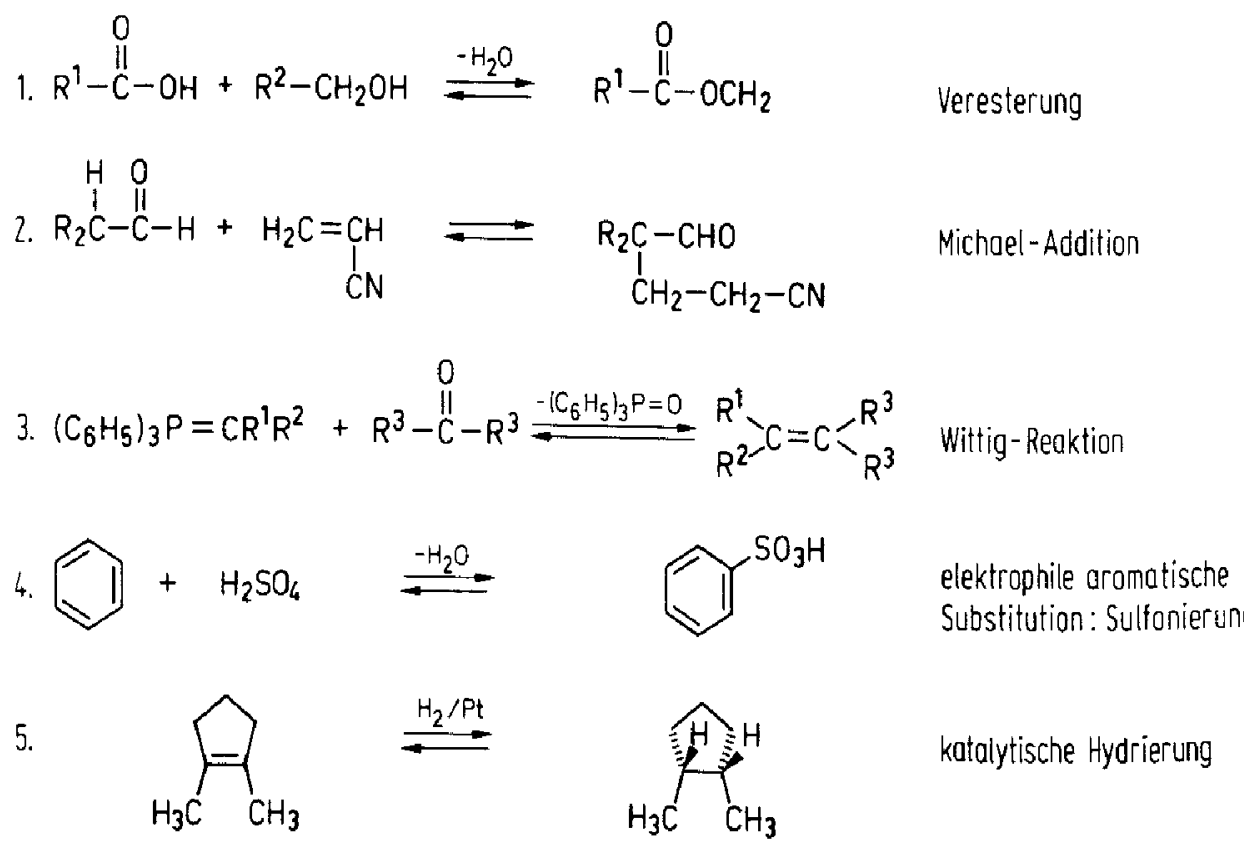

Abb. 4.15 Einige ausgewählte Synthesewege (1-5).

${ }^{1}$ G. Wittig, Deutscher Nobelpreisträger für Chemie (1979) 


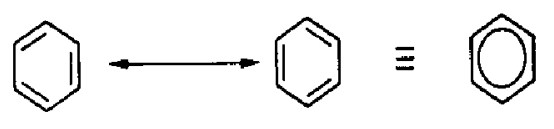

Benzol, $\mathrm{C}_{6} \mathrm{H}_{6}$

(a)

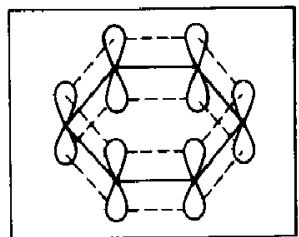

(b)<smiles>[B]c1ccccc1I</smiles>

(c)

Abb. 4.16 Benzol, der Grundkörper der aromatischen Verbindungen: (a) Mesomere Grenzstrukturen des Benzols. (b) Schematische Darstellung des delokalisierten Elektronensystems bzw. der $\pi$-Wolke oberhalb und unterhalb der Ringebene. (c) Mesomere Grenzstrukturen eines Benzolderivates.

sierten Elektronen bilden eine $\pi$-Wolke oberhalb und unterhalb der Ringebene (Abb. 4.16b).

Tatsächlich zeigen experimentelle Untersuchungen, daß keine alternierenden Einfach- bzw. Doppelbindungen vorliegen, wie man es für ein „Cyclohexatrien“ erwarten müßte. Beim Vergleich der Hydrierwärmen (bei der katalytischen Hydrierung der Doppelbindungen freiwerdende Energie) zeigt sich Benzol um $124 \mathrm{~kJ} / \mathrm{mol}$ stabiler als das hypothetische Cyclohexatrien (berechneter Wert bei lokalisierten Doppelbindungen). Diese Stabilisierung wird durch die Delokalisierung der sechs $\pi$-Elektronen erreicht und auch als Resonanzenergie des Benzols bezeichnet. Das zugrundeliegende allgemeine Phänomen heißt aus historischen Gründen Aromatizität. Das Benzolmolekül bildet ein reguläres Sechseck, bei dem die Länge der aromatischen C-C-Bindungen zwischen denen einer Einfach- und einer Doppelbindung liegen. Man kann diese Struktur durch zwei gleichwertige Grenzstrukturen von Cyclohexatrien wiedergeben (Abb. 4.16a, c; Mesomerie). Diese Tatsachen haben erhebliche Konsequenzen für die chemischen und spektroskopischen Eigenschaften dieses Strukturtyps und somit für die große Zahl aller aromatischen Verbindungen.

\subsubsection{Stereochemie}

Die Konstitution einer Verbindung mit gegebener Summenformel (z. B. $\mathrm{C}_{n} \mathrm{H}_{2 n+2}$ ) wird durch die Art und Aufeinanderfolge der Bindungen der beteiligten Atome festgelegt, wobei die Zahl der theoretisch möglichen Konstitutionsisomeren mit steigender Kohlenstoffzahl ungeheuer gro $B$ wird. $\mathrm{C}_{20} \mathrm{H}_{42}$, ein einfacher Kohlenwasserstoff, erlaubt bereits 366319 Isomere. Der höchste Kohlenwasserstoff, von dem alle Konstitutionsisomeren dargestellt wurden, ist $\mathrm{C}_{9} \mathrm{H}_{20}$ mit 35 nichtäquivalenten Konstitutionen. Man unterscheidet zum Beispiel Stellungsisomere, Tautomere, E/Z-Isomere u. a. (Abb. 4.17).

In der Organischen Chemie wird neben Konstitution insbesondere der Begriff 
Stellungsisomere<smiles>CCCC(C)C</smiles><smiles>CCC(C)CC</smiles>

Toutomere<smiles>CC(=O)CC(C)=O</smiles><smiles>CC(=O)C=C(C)O</smiles>

E/Z- Isomere<smiles>O=C(O)C=CC(=O)O</smiles>

Abb.4.17 Beispiele isomerer Verbindungen.

„Struktur" benutzt, den wir, wie in der Biochemie und Röntgenstrukturanalyse üblich, gleichbedeutend mit der dreidimensionalen Anordnung der Atome (Konfiguration, Konformation) verstehen wollen: Konformationsisomere besitzen die gleiche Konfiguration, d.h. eine identische räumliche Verknüpfung aller Bindungspartner. Sie sind durch Drehung um Einfachbindungen (ca. $12 \mathrm{~kJ} / \mathrm{mol}$ ) ineinander überführbar. Abb. 4.18 zeigt die möglichen Anordnungen von Ethan und die stabilen Konfigurationen anhand des Potentialverlaufs als Funktion der Drehung um die zentrale $\mathrm{C}$-C-Einfachbindung.

Die Stereochemie erläutert insbesondere den dreidimensionalen Aufbau von Verbindungen und ist bei der Beschreibung und Analytik eng mit Betrachtungen zur Symmetrie verknüpft.

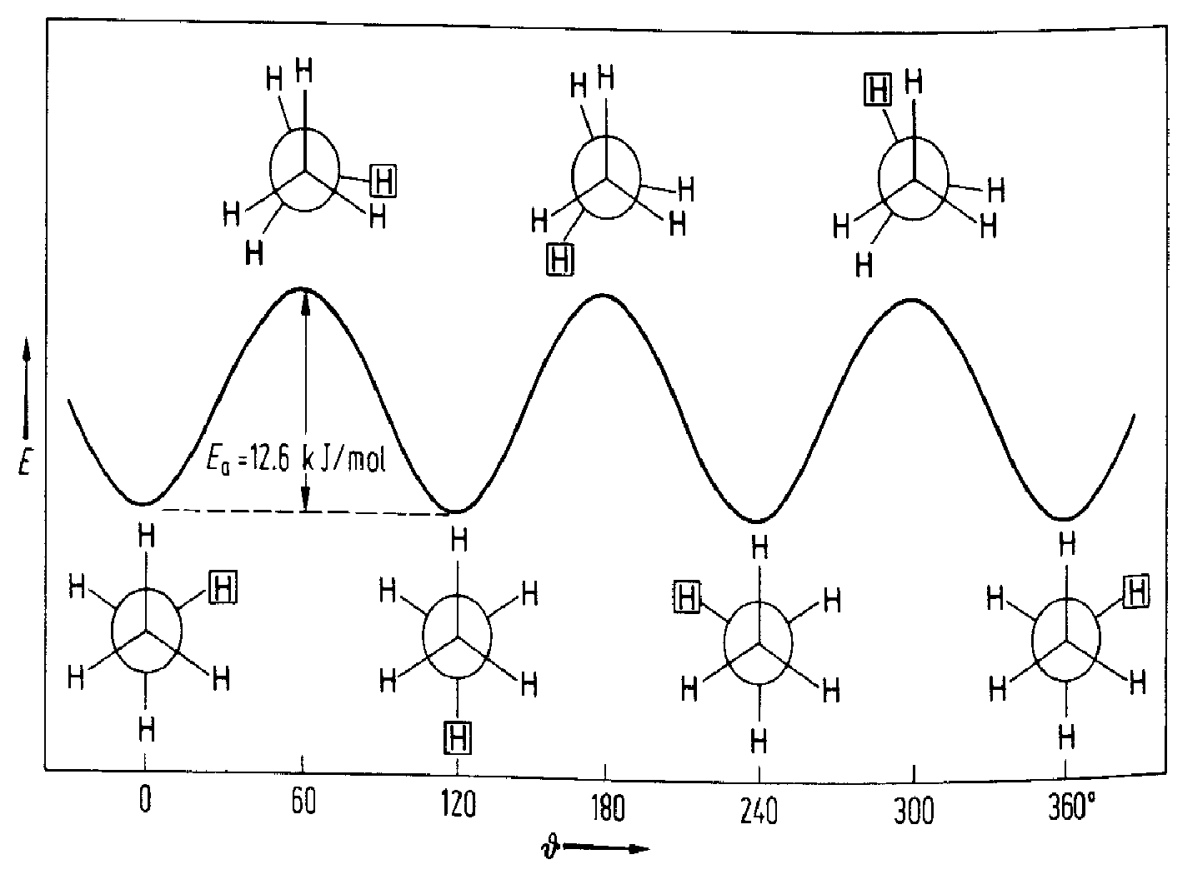

Abb.4.18 Konformationsänderung durch Rotation um die Kohlenstoff-Kohlenstoff-Bindung: Torsionspotentialprofil des Ethans $\left(\mathrm{C}_{2} \mathrm{H}_{6}\right.$; Projektion, die vordere $\mathrm{CH}_{3}$-Gruppe behält ihre Position) als Funktion des Drehwinkels $\vartheta$. 


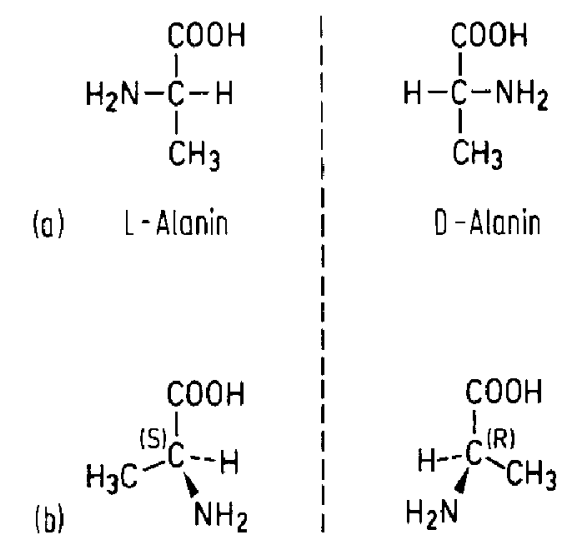

Abb. 4.19 Chiralität: Enantiomere $\alpha$-Aminosäuren (Alanin). (a) Zweidimensionale FischerProjektion; D,L-Nomenklatur. (b) Dreidimensionale Darstellung; R/S-Nomenklatur (beim abgebildeten Alanin sind jeweils die D- und (R)-Form bzw. die L- und (S)-Form identisch).

Die rasante Entwicklung der organischen Stereochemie hat in den letzten Jahren viele neue Begriffe entstehen lassen, die sämtliche beobachteten und zu erläuternden Phänomene wissenschaftlich möglichst eindeutig und einheitlich beschreiben sollen [3]. Besonders wichtig ist der Begriff der Chiralität, denn es ist lange bekannt, daß es weitgehend chirale Moleküle sind, auf denen die komplexe Chemie des Lebens beruht. Solche chiralen Moleküle können in zwei nicht identischen stereoisomeren Formen - den Enantiomeren - auftreten, die sich wie Bild und Spiegelbild verhalten (Konfigurationsisomere; R/S- bzw. D/L-Nomenklatur; vgl. Abb.4.19). In der Natur kommt in der Regel jedoch nur eine enantiomere Form vor, wie z. B. die L-Aminosäuren, die insbesondere zum Aufbau der Proteine dienen.

Da Enantiomere mit einer chiralen Umgebung jeweils unterschiedlich in Wechselwirkung treten, ist verständlicherweise auch die biologische Aktivität der beiden Enantiomeren von Natur- und Wirkstoffen in der Regel verschieden. Die Abb. 4.20 zeigt einige dafür eindrucksvolle Beispiele. So wirkt beim Contergan, das als Gemisch aus gleichen Mengen beider Enantiomerer (Racemat) verwendet wurde, nur ein Antipode extrem teratogen, während das Spiegelbild keinerlei Mißbildungen hervorruft!

Da es schon immer ein Hauptziel war, es dem großen Vorbild Natur gleichzutun, hat sich die Methode der sogenannten asymmetrischen Synthese entwickelt. Aber erst in den letzten Jahren haben wir gelernt, gewünschte asymmetrische Reaktionen tatsächlich gezielt unter hoher Stereokontrolle durchzuführen, d. h. die Herstellung möglichst enantiomerenreiner Verbindungen, wie Arzneimittel, Geschmacks- und Riechstoffe, Insektizide, Pheromone etc., in vitro zu erreichen. Wie die Abb. 4.21 am Beispiel der Addition einer magnesiumorganischen Verbindung an die prochirale Carbonylgruppe eines Aldehyds lehrt, mußten Differenzen für die Aktivierungsenergien der zu den beiden Spiegelbildern führenden Konkurrenzreaktionen von $\Delta \Delta \mathrm{G}^{*}>10 \mathrm{~kJ} / \mathrm{mol}$ erreicht werden, um nahezu enantiomerenreine Produkte zu erhalten. Dies wurde insbesondere durch die Nutzung preiswerter Naturstoffe (chiral pool) als enantiomerenreine Hilfsreagentien (* in Abb. 4.21: Chiral pool-Verbindungen wie Weinsäure, Kohlenhydrate, Alkaloide etc. übertragen als Katalysatoren chirale Information) in Verbindung mit metallorganischen Präparaten und Reaktions- 


$$
\text { 5-Konfiguration }
$$<smiles>NC(=O)CC(N)(N)C(=O)O</smiles>

bitter<smiles></smiles>

Krampfantall<smiles>O=C1CC[C@H](N2C(=O)c3ccccc3C2=O)C(=O)N1</smiles>

extrem terologen<smiles>C=C(C)C1CC=C(C)CC1</smiles>

Geruch nach Zitronen<smiles>CC(C)(S)[C@H](N)C(=O)O</smiles>

Antiarthriticum<smiles>N[C@@H](Cc1cc(I)c(Oc2cc(I)c(O)c(I)c2)c(I)c1)C(=O)O</smiles>

Schilddrüsen - Hormon<smiles>CC(C)NC[C@H](O)COc1cccc2ccccc12</smiles>

$\beta$-Blocker

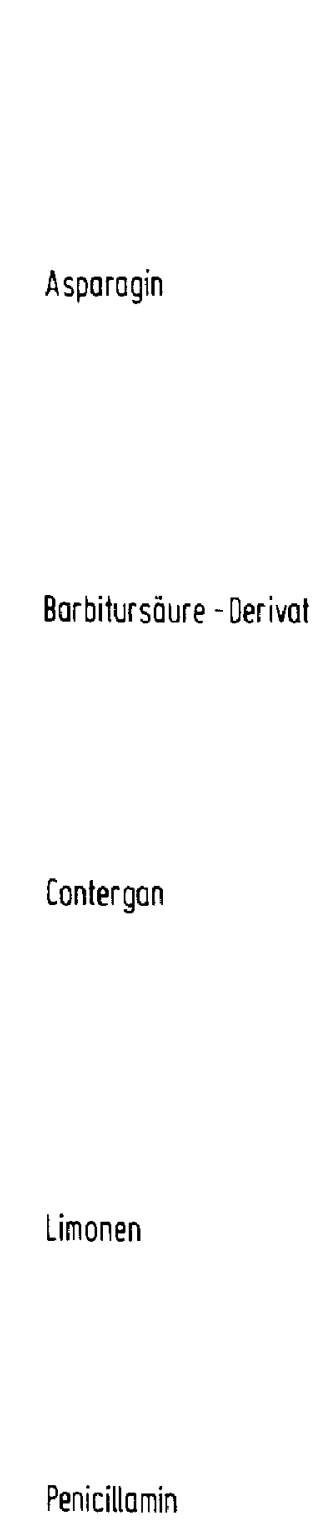

R-Konfiguration<smiles>NC(=O)C[C@H](N)C(=O)O</smiles>

sün

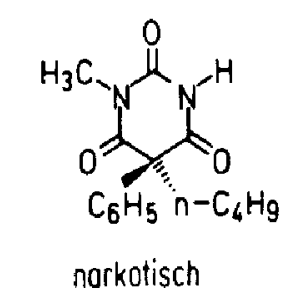<smiles>C[C@@]1(N2C(=O)c3ccccc3C2=O)CCC(=O)NC1=O</smiles>

keine Mißbildungen<smiles>C=C(C)C1CC=C(C)CC1</smiles>

Geruch nach Orangen<smiles>CC(C)(S)[C@H](N)C(=O)O</smiles>

extrem toxisch<smiles>N[C@@H](Cc1cc(I)c(Oc2cc(I)c(O)c(I)c2)c(I)c1)C(=O)O</smiles>

Antihypocholesterinicum<smiles>CC(C)NC[C@H](O)COc1cccc2ccccc12</smiles>

Contraceptivum

Abb.4.20 Unterschiede der biologischen Aktivität einiger spiegelbildisomerer Natur- bzw. Wirkstoffe. 

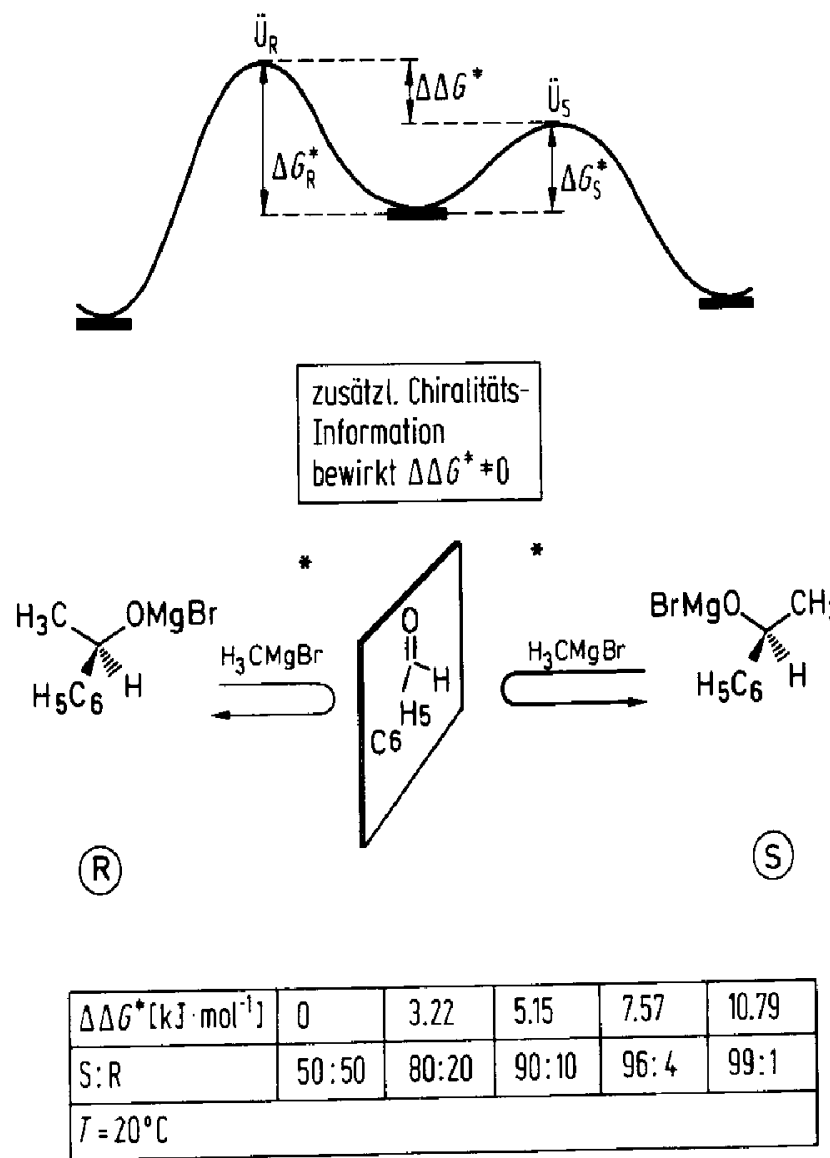

Abb. 4.21 Erzeugung eines neuen Chiralitätszentrums durch Addition einer magnesiumorganischen Verbindung $\left(\mathrm{CH}_{3} \mathrm{MgBr}\right)$ an einen prochiralen Aldehyd $\left(\mathrm{C}_{6} \mathrm{H}_{5} \mathrm{CHO}\right)$ in Gegenwart einer zusätzlichen Chiralitätsinformation *. Der Zusammenhang des erreichbaren Überschusses eines Enantiomeren ( $\mathrm{R}$ oder $\mathrm{S}$ ) ist in Abhängigkeit von $\Delta \Delta G^{*}$ angegeben.

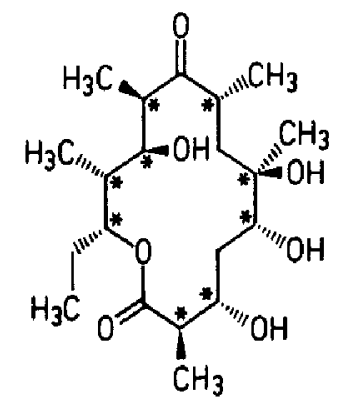

Abb. 4.22 Struktur des Macrolid-Antibiotikums Erythronolid B, das trotz der stereochemischen Komplexität mit modernen präparativen Methoden synthetisiert werden konnte (die Chiralitätszentren sind mit * bezeichnet).

führung bei tiefstmöglichen Temperaturen erreicht, wobei sich als Metalle u.a. $\mathrm{Mg}$, $\mathrm{Ti}, \mathrm{Li}, \mathrm{B}, \mathrm{Al}, \mathrm{Zr}$ und $\mathrm{Sn}$ bewährt haben.

Trainings- und Wettkampfplatz für diese neuen Verfahren, die viel zum Verständnis mechanistischer Abläufe beigetragen haben und von denen man speziell auf dem 
Wirkstoffsektor (Pharma) noch einiges erwarten kann, sind komplexe Naturstoffsynthesen. Die entscheidende Analysemethode ist sehr oft die Kernresonanzspektroskopie (NMR, vgl. Abschn. 5.21).

Die vielen Chiralitätszentren $(*)$ des Erythronolid B (Abb. 4.22), die unter hoher Stereokontrolle u. a. von der Arbeitsgruppe des Nobelpreisträgers Woodward aufgebaut werden konnten, sollen als eindrucksvolles Beispiel für die Leistungsfähigkeit der stereokontrollierten Synthese dienen [4].

Neuere Entwicklungen befassen sich mit der gezielten Synthese von (Makro-) Molekülen, die Hohlräume mit definierter Geometrie aufweisen. Diese Arbeiten wurden 1987 mit dem Nobelpreis für Chemie ausgezeichnet [5].

\subsubsection{Strukturaufklärung}

Der Begriff Struktur beinhaltet zugleich das Wissen um die Konstitution, die Konfiguration und die Konformation der untersuchten Verbindung. Das Hantieren mit Strukturen gehört für den Chemiker und Molekülphysiker zum alltäglichen Denken und Arbeiten. So versteht er die Fragen nach dem „Aufbau der Materie“ letztlich vor allem auch als das Erkennen der molekularen Struktur (natürlich auch Suprastrukturen [5]), denn erst auf der Basis des Wissens um die Struktur kann moderne (auch industrielle) Chemie als gezielte chemische Umsetzung geplant werden.

Ein vorrangiges Problem der Analytik besteht in der Frage nach der Reinheit (Einheitlichkeit) der zu untersuchenden Probe. Häufig müssen z. T. extrem aufwendige Auftrennungs- und Reinigungsverfahren vorangestellt werden wie Gaschromatographie, Flüssigkeitschromatographie (HPLC), Ionenchromatographie, Elektrophorese etc. In vielen Fällen gelingt eine eindeutige Charakterisierung aber auch im Gemisch.

Viele sehr erfolgreiche klassisch chemische Verfahren zur Strukturermittlung sind bekannt, die das spezifische Verhalten bei chemischen Reaktionen beschreiben (Brennbarkeit, Verhalten gegenüber Säuren, Basen, Reduktions-, Oxidationsmitteln etc.). Oder man nutzt charakteristische Eigenschaften von Verbindungen wie Schmelzpunkt, Siedepunkt, Brechzahl, Löslichkeit, Härte, Leitfähigkeit etc., die sich messen oder beobachten lassen, ohne zu stofflichen Veränderungen zu führen. Die enorme Entwicklung im Apparatebau und speziell in der Datenerfassung und -verarbeitung hat allerdings zum Aufschwung der instrumentellen Analytik beigetragen und dominiert heute eindeutig die Strukturaufklärung. Zu nennen sind die praktisch ausschließlich von Physikern [6] ${ }^{1}$ entwickelten Methoden und modernen instrumentellen Techniken (vgl. Abschn. 5.2) wie NMR-, IR-, UV- und ESR-Spektroskopie, Massenspektrometrie, Elektronenstreuung, Röntgenbeugung etc.; vor allem die Kernresonanzspektroskopie (z. B. ${ }^{1} \mathrm{H}-,{ }^{19} \mathrm{~F}-,{ }^{31} \mathrm{P}-\mathrm{NMR}$ ) hat von dieser Entwicklung profitiert und den Nachteil der relativ geringen Empfindlichkeit inzwischen sogar bei der Untersuchung von ,problematischen“ Kernen $\left({ }^{13} \mathrm{C},{ }^{15} \mathrm{~N},{ }^{17} \mathrm{O},{ }^{23} \mathrm{Na}\right.$ etc. $)$ weitgehend überwunden (siehe Abb. 5.1-5.5). Man nutzt die magnetischen Eigenschaften der Kerne, die einen Drehimpuls aufweisen. Nach der Anregung durch Bestrahlen mit Energie im Radiofrequenzbereich in einem möglichst homogenen Magnetfeld

${ }^{1}$ R. R. Ernst, Nobelpreisträger für Chemie (1991) 
(Supraleiter ermöglichen die für leistungsfähige Verfahren notwendigen Magnetfeldstärken von $10 \mathrm{~T}$ und mehr) erhält man durch Beobachtung der induzierten Magnetisierung und des Relaxationsverhaltens Aussagen über die Chemische Verschiebung und zu den „Spinsystemen“ gekoppelter Kerne und damit entscheidende Informationen zur Strukturermittlung.

Moderne NMR-Experimente [7] ermöglichen mittels komplexer Pulsfolgen sogenannte 2D NMR-Experimente, die z. B. eine einfache und übersichtliche Korrelation von Kopplungspartnern erlauben und damit präzise Aussagen über Nachbarschaften und stereochemische Anordnungen auch in komplexen Molekülen zulassen (vgl. Abschn. 4.3.2).

Anwendungen haben sich neuerdings auch im Bereich der medizinischen Diagnostik durch den Bau von NMR-Tomographen ergeben. In vielen Fällen kann jetzt auf die mit ionisierender Strahlung verbundene Röntgentomographie verzichtet werden, da im NMR häufig gleichwertige und meistens zusätzliche Ergebnisse sichtbar werden (stark wasserhaltige Gewebe liefern differenzierte Bilder; siehe Abb. 5.2.1.6).

Abschließend muß darauf hingewiesen werden, daß der anschauliche Begriff der Struktur heute teilweise in Frage gestellt wird. Durch Anregungen der Moleküle in hohe Vibrationszustände ist es möglich, Anordnungen der Atome im Molekül zu erreichen, die den einfachen Oktettregeln stark widersprechen. Durch Tunneln kann das Molekül resonanzartig mehrere Anordnungen der Atome erreichen und die geometrische Struktur als eindeutige Beschreibung ad absurdum führen.

\subsubsection{Beispiele interessanter Strukturen}

Neben der von direkten Anwendungsaspekten geleiteten Forschung und Entwicklung spielt nach wie vor die reine Grundlagenforschung eine auch von der Industrie geschätzte, weitgehend gleichberechtigte Rolle. Zu den Lieblingsthemen gehört die Synthese attraktiver bzw. ungewöhnlicher Strukturen, wobei das Ungewöhnliche sowohl durch die Komplexität der Struktur als auch durch die Neuartigkeit begründet sein kann - oder aber durch die Tatsache, daß man diese Struktur vorher für sehr instabil gehalten hat, jetzt aber durch spezielle Synthesetricks erzeugen und durch geeignete Substituenten stabilisieren bzw. durch ausgefeilte Isolations-(Matrix) oder Abfangverfahren belegen kann.

Hierzu existieren viele heute bereits klassische Beispiele (Edelgasverbindungen, Borhydride, Metallcarbonyle ...), sowie moderne neue Beispiele (Tetrahedran, Dodecahedran, Hexaazakekulen etc.; Abb. 4.23. Diese Auflistung versteht sich als subjektive Auswahl ohne Bewertung der Qualität und ohne Anspruch auf Vollständigkeit)

Auf diesen zunächst ausschließlich von der Grundlagenforschung geprägten Forschungsgebieten entwickeln sich immer wieder neue, innovative und ausgefallene Ideen und Strategien, ohne Rücksicht auf das sonst meist vorgegebene Korsett möglicher Anwendungen. Kreativität ist hier ganz besonders gefordert, ein enormer Lerneffekt häufig der Lohn!

Die Suche nach physiologisch wirksamen Stoffen (bioactive compounds) stellt oft ein Bindeglied zwischen dieser Form der Grundlagenforschung und möglichen Anwendungen dar: Hier sind $z$. B. Leitstrukturen gefragt, an denen man sich erfahrungs- 


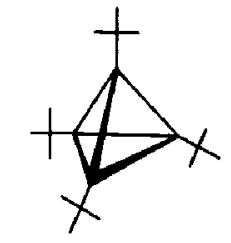

(a)

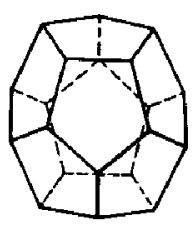

(b)<smiles>c1cc2ncc1-c1nc3c(cc1CC2)CCc1cc2c(nc1-3)-c1nc3c(cc1CC2)CCc1cc2c(nc1-3)-c1nc3c(cc4cc(c(n1)CC4)CC2)CCC3</smiles>

(c)

Abb. 4.23 Drei Beispiele attraktiver bzw. ungewöhnlicher Strukturen: (a) Tetra-tert-butyltetrahedran, (b) Dodecahedran, (c) Dodecahydrohexaazakekulen.

gemäß orientieren kann. Diese findet man häufig in altbewährten oder auch neuartigen Naturstoffstrukturen, die Total- bzw. Partialsynthesen auf diesem Gebiet stimulieren, aber auch in völlig neuartigen und vielleicht ungewöhnlichen Verbindungsklassen. Neben neuen präparativen Methoden werden insbesondere die ungemein vielfältigen Formen der Stereochemie zum Hauptthema und eben dies hat die Kontrolle der Stereochemie im Zuge einer chemischen Reaktion zu einem hochaktuellen Forschungsgebiet gemacht.

Auch in vielen anderen Bereichen der Chemie haben sich ungewöhnliche Entwicklungen vollzogen. Während früher die Suche nach Anwendungen für vorhandene Produkte die Forschung und Entwicklung prägte, steht heute und in Zukunft immer mehr das Entwerfen maßgeschneiderter Stoffe nach Bedürfnissen und Vorstellungen der Verbraucher im Vordergrund. Schlagzeilen machen neben den keramischen Supraleitern insbesondere neuartige Polymere. Als wesentliche Vorzüge von Kunststoffen galt bislang, daß sie preiswert, haltbar, leicht und vielseitig anwendbar sind. Ihre ausgezeichneten Isolatoreigenschaften sind lange bekannt. Neuartige Polymere wie mit Iod dotiertes Polyacetylen, Polypyrrol oder Polyanilin leiten den elektrischen Strom aber schon vergleichbar gut wie Metalle [8]. Trotz einiger Kinderkrankheiten eröffnen sich neue Einsatzmöglichkeiten für diese Vielzweckwerkstoffe (Polymerakkumulator, Elektronik, Klebstoffe etc.).

\section{Literatur}

\section{Weiterführende Literatur}

Atkins, P. W., Physikalische Chemie, Verlag Chemie, Weinheim, 1987

Beyer, H., Walter, W., Lehrbuch der Organischen Chemie, Hirzel, Stuttgart, 1988

Buddrus, J., Grundlagen der Organischen Chemie, de Gruyter, Berlin, New York, 1989 
Dickerson, R.E., Gray, H. B., Darensbourg, M.Y., Darensbourg, D.J., Prinzipien der Chemie, de Gruyter, Berlin, New York, 1988

Günther, H., NMR-Spektroskopie, Thieme, Stuttgart, New York, 1983

Haiduc, I., Zuckerman, J. J., Basic Organometallic Chemistry, de Gruyter, Berlin, New York, 1985

Holleman, A.F., Wiberg, N., Lehrbuch der Anorganischen Chemie, de Gruyter, Berlin, New York, 1985

Weissermel, K., Arpe, H.J., Industrielle Organische Chemie, Verlag Chemie, Weinheim, 1987

\section{Zitierte Publikationen}

[1] International Union of Pure and Applied Chemistry, Nomenclature of Organic Chemistry, Sections A, B, C, D, E, F und H, 1979 Edition, Pergamon Press

[2] Woodward, R. B., Hoffmann, R., Die Erhaltung der Orbitalsymmetrie, Angew. Chem. 81, 797-870, 1969

[3] Masamune, S., Choy, W., Petersen, J.S., Sita, L.R., Doppelte Stereodifferenzierung und eine neue Strategie zur Stereokontrolle in der Organischen Synthese, Angew. Chem. 97, $1-78,1985$

[4] Woodward, R. B. et al., Asymmetric Total Synthesis of Erythromycin, J. Am. Chem. Soc. $103,3215-3217,1981$

[5] Cram, D. J., Von molekularen Wirten und Gästen sowie ihren Komplexen, Angew. Chem. 100, 1041-1056, 1988

[6] Aue, W.P., Bartholdi, E., Ernst, R. R., Two-Dimensional Spectroscopy, Application to Nuclear Magnetic Resonance, J. Chem. Phys. 64, 2229-2246, 1976

[7] Benn, R., Günther, H., Moderne Pulsfolgen in der hochauflösenden NMR-Spektroskopie, Angew. Chem. 95, 381-411, 1983

[8] Kaner, R. B., McDiarmid, A.G., Elektrisch leitende Kunststoffe, Spektrum der Wissenschaft, April 1988, 54-59. 